March 2000

DFUB 00-05

Version 1

hep-th/0003230

\title{
3-point functions of universal scalars in maximal SCFTs at large $N$
}

\author{
by \\ Fiorenzo Bastianelli and Roberto Zucchini \\ Dipartimento di Fisica, Università degli Studi di Bologna \\ V. Irnerio 46, I-40126 Bologna, Italy \\ and \\ INFN, Sezione di Bologna
}

\begin{abstract}
We compute all 3-point functions of the "universal" scalar operators contained in the interacting, maximally supersymmetric CFTs at large $N$ by using the AdS/CFT correspondence. These SCFTs are related to the low energy description of M5, M2 and D3 branes, and the common set of universal scalars corresponds through the AdS/CFT relation to the fluctuations of the metric and the magnetic potential along the internal manifold. For the interacting $(0,2) \mathrm{SCFT}_{6}$ at large $N$, which is related to M5 branes, this set of scalars is complete, while additional non-universal scalar operators are present in the $d=4, \mathcal{N}=4$ super Yang-Mills theory and in the $\mathcal{N}=8 \mathrm{SCFT}_{3}$, related to D3 and M2 branes, respectively.
\end{abstract}

Keywords: String Theory, Conformal Field Theory, Geometry.

PACS no.: 0240, 0460, 1110. 


\section{Introduction}

The low energy dynamics of non-dilatonic superstring/M-theory branes identify an interesting class of interacting CFT possessing maximal supersymmetry [1]. These SCFT should presumably be given a description in terms of the collective coordinates of the branes. This is well known for a system of $N$ coinciding D3 branes, whose collective degrees of freedom span the $d=4, \mathcal{N}=4 U(N)$ super vector multiplet and whose infrared dynamics is described precisely by the corresponding super Yang-Mills theory [2]. A similar explicit description for a set of $N$ coinciding M5 or M2 branes is unknown. Indeed, it is only known that the collective coordinates of a single M5 brane form a $d=6, \mathcal{N}=(0,2)$ free tensor multiplet (a 2-form with selfdual field strength, 5 scalars and 2 Weyl fermions) $[3,4]$ and that those of a single M2 brane form a $d=3, \mathcal{N}=8$ free scalar multiplet ( 8 bosons and 8 Majorana fermions) [5]. Interacting SCFTs describing the collective coordinates of $N$ coinciding M5 or M2 branes remain instead quite mysterious. However, superstring/M-theory predicts the existence of these models [6-8] and, in fact, suggests a full ADE classification. A concrete handle on the problem is provided by the AdS/CFT duality conjecture, which relates these SCFT in $d=3,4,6$ to superstring/M-theory on $\mathrm{AdS}_{4,5,7} \times \mathrm{S}_{7,5,4}$ [9-11]. Specifically, at large $N$, one can approximate the superstring/Mtheory by the corresponding classical supergravity. Then, the latter may be used to obtain informations on the strong coupling limit of the related interacting SCFTs and, in the case of $\mathrm{SCFT}_{3,6}$, also to get important clues about their mysterious lagrangian formulation. The AdS/CFT duality conjecture has been tested extensively in the literature (see ref. [12] and references therein) and used to compute 3- and 4-point functions of some chiral operators (a partial list consists of refs. [13-24]).

In this paper, we continue our analysis of the AdS/CFT correspondence presented in [22-24], and address the problem of computing the 3 point functions for a set of scalar operators which are present in all of the above mentioned theories at large $N$. In fact, there are three families of such scalars, to be denoted by $\mathcal{O}^{s}, \mathcal{O}^{f}, \mathcal{O}^{t}$, which are in correspondence with the metric and the magnetic potential fluctuations along the internal manifold (the metric contributing with two Kaluza-Klein families due to its splitting into a trace and a traceless part). They form a kind of "universal" scalar sector common to all the nondilatonic branes, which is reminiscent of the NS-NS universal sector present in the spectrum of the various closed superstrings. At large $N$, all but a finite number of the supersymmetric short multiplets contain precisely one scalar $\mathcal{O}^{s}, \mathcal{O}^{f}, \mathcal{O}^{t}$, with $\mathcal{O}^{s}$ being the chiral primary. Their conformal dimensions are given by

$$
\Delta^{s}=\Delta, \quad \Delta^{f}=\Delta+2, \quad \Delta^{t}=\Delta+4,
$$


where

$$
\Delta=\frac{n+1}{D-n-3} k,
$$

$n$ is the dimension of the brane, $D$ is the space time dimension and $k \geq 4$ is an integer characterizing the multiplet. The exceptional multiplets, corresponding to the values $k=$ 2,3, do not contain the operator $\mathcal{O}^{t}$. For the M5 brane case, this set of scalars is complete at large $N$, as all other operators have a non trivial tensorial character. On the other hand, for the M2 and D3 branes, additional non-universal scalars are present in the spectrum (see, e.g. the tables in $[25,26]$ ).

The simultaneous treatment of the universal sector is made possible by the use of the general gravitational model introduced in [23], which was shown later in [24] to correctly reproduce the universal scalar self couplings also in the case of the dyonic D3 brane (where the full lagrangian of type IIB supergravity contains a 4-form with selfdual field strength).

Thus, in the following, we briefly review our general gravitational model and present the result for all of the 3-point couplings of the universal scalars. As a check, we have carried out the computation in two independent ways, by expanding the action at the cubic order in the scalar fields and by studying the quadratic corrections to the scalar equations of motion. Of course, we obtained the same final result. Then, the application of AdS/CFT duality allows us to obtain the announced universal scalar 3-point functions. We tried to cast the resulting expressions in a way which may suggest a group theoretic interpretation. Finally, we present some technical details on tensor spherical harmonics and their integrals in an appendix.

\section{Identification of the universal scalar sector}

According to the AdS/CFT duality principles [9-11], the low energy world volume CFT of $N$ coincident M5, M2, D3 branes at large $N$ is described by $D=11, D=11$, $D=10$ type IIB classical supergravity compactified on $\mathrm{AdS}_{7} \times \mathrm{S}_{4}, \mathrm{AdS}_{4} \times \mathrm{S}_{7}, \mathrm{AdS}_{5} \times \mathrm{S}_{5}$, respectively. The CFT scalar fields, whose three point functions we want to compute, are related by duality in a precise way to certain fluctuations of the AdS bosonic supergravity fields around a maximally supersymmetric Freund-Rubin background [27]. In ref. [23, 24], it has been shown that the dynamics of these fluctuations up to third order is governed by a gravitational action that has the same form for all the three types of branes mentioned above and is, therefore, universal. Let us describe and justify the model briefly.

Space time $M_{D}$ is $\operatorname{AdS}_{D-2-p} \times \mathrm{S}_{2+p}$. The relevant fields are the metric $g$ and the

$1+p$ form field $A_{1+p}$ with field strength $F_{2+p}=d A_{1+p}{ }^{1}$. The Freund-Rubin background

1 We denote form degree on $M_{D}$ by a subfix, e. g. $\omega_{r}$ is a $r$ form on $M_{D}$. 
$\bar{g}, \bar{A}_{1+p}$ is such that $\bar{g}$ is factorized and $\bar{F}_{2+p}=\bar{F}_{(0,2+p)}$. The relevant bosonic fluctuations of $g$ and $A_{1+p}$ around the background are such that $g$ remains factorized and $F_{2+p}=$ $\bar{F}_{(0,2+p)}+d a_{(0,1+p)}{ }^{2}$. The action is given by

$$
\begin{gathered}
I=\frac{1}{4 \kappa^{2}} \int_{\mathrm{AdS}_{D-2-p} \times \mathrm{S}_{2+p}}\left[R(g) *_{g} 1-F_{2+p} \wedge *_{g} F_{2+p}\right], \\
g=g^{\prime} \oplus g^{\prime \prime}, \quad F_{2+p}=\bar{F}_{(0,2+p)}+d a_{(0,1+p)} .
\end{gathered}
$$

The important cases are those for which $(D, p)=(11,2),(11,5),(10,3)$.

For the M5 theory $((D, p)=(11,2))$, the above action is obtained directly from that of the bosonic sector of $D=11$ supergravity by noting that, for the fluctuation considered here, the Chern-Simons term vanishes identically.

For the M2 theory $((D, p)=(11,5))$, the situation is slightly more complicated. Using the standard 3-form formulation of $D=11$ supergravity is inconvenient as the relevant scalar fluctuation contained in the fluctuation $a_{(3,0)}$ of $A_{3}$ comes about as the solution of the constraint

$$
d *_{\bar{g}^{\prime}} a_{(3,0)}=0
$$

entailed by gauge fixing at quadratic level, which is difficult to implement in an off-shell fashion. This problem can be solved by means of a standard dualization trick, whereby the 3 -form $A_{3}$ is replaced by a 6 -form $A_{6}$ such that $F_{7}=*_{g} F_{4}$. Since the Chern-Simons term vanishes also in this case for the fluctuation considered here, the resulting action takes the simple form (1.1). However, in the dual formulation, the relevant scalar fluctuation is contained in the fluctuation $a_{(0,6)}$ of $A_{6}$ and can thus be treated in an off-shell fashion in a way completely analogous to that of the M5 brane.

For the D3 theory $((D, p)=(10,3))$, the relevant fields are the metric $g$ and the IIB Ramond-Ramond 4 form field $A_{4}$ with selfdual field strength $F_{5}^{s d}=d A_{4}$

$$
F_{5}^{s d}=*_{g} F_{5}^{s d}
$$

The selfdual Freund-Rubin background $\bar{g}, \bar{A}_{4}$ is such that $\bar{g}$ is factorized and $\bar{F}_{5}^{s d}=$ $2^{-\frac{1}{2}}\left(\bar{F}_{(0,5)}+*_{\bar{g}} \bar{F}_{(0,5)}\right)$. The relevant fluctuations of $g$ and $A_{4}$ around the background are

2 We say that a metric $g$ on $M_{D}$ is factorized if $g$ has the block structure $g=g^{\prime} \oplus g^{\prime \prime}$, where $\bar{g}^{\prime}, \bar{g}^{\prime \prime}$ are metrics on $\operatorname{AdS}_{D-2-p}, \mathrm{~S}_{2+p}$, respectively. Similarly, we denote form degree on the factors $\operatorname{AdS}_{D-2-p}, \mathrm{~S}_{2+p}$ by a pair of suffixes, e. g. $\omega_{(r, s)}$ denotes a $r+s$ form on $M_{D}$ that is a $r$ form on $\mathrm{AdS}_{D-2-p}$ and a $s$ form on $\mathrm{S}_{2+p}$. 
such that $g$ is factorized, as usual, and $F_{5}^{s d}=\bar{F}_{5}^{s d}+d a_{4}$, where $a_{4}=2^{-\frac{1}{2}}\left(a_{(4,0)}+a_{(0,4)}\right)$. The selfduality equations (1.4) relate the fluctuations $a_{(4,0)}, a_{(0,4)}$ and allow in principle to express $a_{(4,0)}$ in terms of $a_{(0,4)}$. Upon doing this, the resulting field equations can be seen to be equivalent to those obtained from the action (1.1).

The standard $\mathrm{AdS}_{D-2-p} \times \mathrm{S}_{2+p}$ Freund Rubin solution $\bar{g}_{i j}, \bar{A}_{i_{1} \cdots i_{1+p}}$ of the field equations following from the action (1.1) is given by

$$
\begin{array}{rlrl}
\bar{R}_{\kappa \lambda \mu \nu} & =-a^{2}\left(\bar{g}_{\kappa \mu} \bar{g}_{\lambda \nu}-\bar{g}_{\kappa \nu} \bar{g}_{\lambda \mu}\right), & a^{2}=\frac{(1+p)}{(D-2)(D-3-p)} e^{2}, \\
\bar{R}_{\alpha \beta \gamma \delta} & =\bar{e}^{2}\left(\bar{g}_{\alpha \gamma} \bar{g}_{\beta \delta}-\bar{g}_{\alpha \delta} \bar{g}_{\beta \gamma}\right), & \bar{e}^{2}=\frac{(D-3-p)}{(D-2)(1+p)} e^{2} \\
\bar{F}_{\alpha_{1} \cdots \alpha_{2+p}} & =e \bar{\epsilon}_{\alpha_{1} \cdots \alpha_{2+p}},
\end{array}
$$

where $\bar{\epsilon}_{\alpha_{1} \cdots \alpha_{2+p}}$ denotes the standard volume form on the unit sphere and $e$ is an arbitrary mass scale parameterizing the compactification ${ }^{3}$. The other components of the Riemann tensor and the field strength vanish identically.

We expand the action in fluctuations around the background $\bar{g}_{i j}, \bar{A}_{i_{1} \cdots i_{1+p}}$. We parameterize the fluctuations $\delta g_{i j}, \delta A_{i_{1} \cdots i_{1+p}}$ of the fields $g_{i j}, A_{i_{1} \cdots i_{1+p}}$ around the background as in $[28]$

$$
\begin{aligned}
& \delta g_{\alpha \beta}= f_{\alpha \beta}+\bar{\nabla}_{\alpha} n_{\beta}+\bar{\nabla}_{\beta} n_{\alpha}+\left(\bar{\nabla}_{\alpha} \bar{\nabla}_{\beta}-\frac{1}{2+p} \bar{g}_{\alpha \beta} \bar{\nabla}^{\gamma} \bar{\nabla}_{\gamma}\right) q+\frac{1}{2+p} \bar{g}_{\alpha \beta} \pi, \\
& f_{\gamma}^{\gamma}=0, \quad \bar{\nabla}^{\gamma} f_{\gamma \alpha}=0, \quad \bar{\nabla}^{\gamma} n_{\gamma}=0, \\
& \delta A_{\alpha_{1} \cdots \alpha_{1+p}}=(1+p) \bar{\nabla}_{\left[\alpha_{1}\right.} a_{\left.\alpha_{2} \cdots \alpha_{1+p}\right]}+\bar{\epsilon}_{\alpha_{1} \cdots \alpha_{1+p}} \gamma \bar{\nabla}_{\gamma} b, \\
& \bar{\nabla}^{\gamma} a_{\gamma \alpha_{3} \cdots \alpha_{1+p}}=0 .
\end{aligned}
$$

Fluctuations of the other components of $g_{i j}$ and $A_{i_{1} \cdots i_{1+p}}$ can be disregarded as they are independent up to third order from the ones we are interested in and therefore do not contribute to the relevant scalar 3-point couplings.

The gauge can be partially fixed by eliminating those gauge invariances which do not correspond to the usual reparameterization and form gauge invariances from the $\mathrm{AdS}_{D-2-p}$ perspective. This can be done by imposing

$$
\bar{\nabla}^{\beta}\left(\delta g_{\beta \alpha}-\frac{1}{2+p} \bar{g}_{\beta \alpha} \delta g_{\gamma}^{\gamma}\right)=0
$$

3 In this paper, we adopt the following conventions. Latin lower case letters $i, j, k, l, \ldots$ denote $M_{D}$ indices. Late Greek lower case letters $\kappa, \lambda, \mu, \nu \ldots$ denote $\operatorname{AdS}_{D-2-p}$ indices. Early Greek lower case letters $\alpha, \beta, \gamma, \delta, \ldots$ denote $\mathrm{S}_{2+p}$ indices. 


$$
\bar{\nabla}^{\beta} \delta A_{i_{1} \cdots i_{p} \beta}=0,
$$

as shown in [28]. Fixing the gauge entails a number of constraints which must be disposed of as explained in [24].

There are three universal families of $\mathrm{AdS}_{D-2-p}$ scalar fields contained in the fluctuations listed above, which we denote as $f_{I}, s_{I}, t_{I}$. The scalar fields $f_{I}$ are defined by

expanding $f_{\alpha \beta}$ (cfr. eq. (1.7)) with respect to an orthonormal basis $\left\{Y_{I}^{(2)}\right\}$ of symmetric traceless transversal 2-tensor spherical harmonics of $\mathrm{S}_{2+p}$ (cfr. appendix A1)

$$
f_{\alpha \beta}=\sum_{I} f_{I} Y_{I \alpha \beta}^{(2)}
$$

The scalar fields $s_{I}, t_{I}$ are given by linear functionals of $\pi, b$ (cfr. eq. (1.7), (1.8)) non local in $\mathrm{S}_{2+p}$ defined as follows. One expands the scalar fields $\pi, b$ with respect to an orthonormal basis $\left\{Y_{I}^{(0)}\right\}$ of scalar spherical harmonics of $\mathrm{S}_{2+p}$ (cfr. appendix A1)

$$
\pi=\sum_{I} \pi_{I} Y_{I}^{(0)}, \quad b=\sum_{I} b_{I} Y_{I}^{(0)}
$$

and identifies the scalar mass eigenstates as given by [23, 24]

$$
\begin{aligned}
s_{I} & =\frac{1}{2 k+1+p}\left(\frac{1}{2(2+p)(D-3-p)} \pi_{I}+\frac{(-1)^{p}(k+1+p)}{(1+p)(D-2)} e b_{I}\right), \\
t_{I} & =\frac{1}{2 k+1+p}\left(\frac{1}{2(2+p)(D-3-p)} \pi_{I}-\frac{(-1)^{p} k}{(1+p)(D-2)} e b_{I}\right) .
\end{aligned}
$$

It should be kept in mind that the range of the quantum numbers $I$ of $f_{I}$ differs from that of the quantum numbers $I$ of $s_{I}, t_{I}$, as these ranges parameterize orthogonal bases of spherical harmonics of different tensorial rank. However, we shall use the same notation for these different quantum numbers for simplicity, as no confusion is possible.

\section{The cubic action of the universal scalar sector}

From the action (1.1), one can extract the couplings of the scalars $f_{I}, s_{I}$ and $t_{I}$ defined in the previous section. After performing some field redefinitions, one finds that their action to cubic order is given by

$$
I_{[\leq 3]}^{f s t}=\frac{1}{4 \kappa^{2}} \int_{\mathrm{AdS}_{d+1}} d^{d+1} y\left(-\bar{g}_{d+1}\right)^{\frac{1}{2}}\left[\frac{1}{2} \sum_{i} A_{i} \psi_{i}\left(\square-m_{i}{ }^{2}\right) \psi_{i}+\frac{1}{3 !} \sum_{i j k} G_{i j k} \psi_{i} \psi_{j} \psi_{k}\right],
$$


where $d=D-3-p, \square$ denotes the d'Alembertian on $\operatorname{AdS}_{d+1}$ and the index $i=\{I, a\}$ contains a flavor index $a$ for the $(f, s, t)$ types of fields, i.e. $\psi_{i}=\psi_{I}^{a}=\left(f_{I}, s_{I}, t_{I}\right)$. The various constants appearing in the actions are given by the following expressions.

$$
\begin{aligned}
& A_{I}^{f}=\frac{1}{2} z_{I} \bar{e}^{-2-p}, \\
& A_{I}^{s}=\frac{2 \nu k(k-1)(2 k+1+p)}{k+\gamma_{s}} z_{I} \bar{e}^{-2-p}, \\
& A_{I}^{t}=\frac{2 \nu(k+1+p)(k+2+p)(2 k+1+p)}{k+\gamma_{t}} z_{I} \bar{e}^{-2-p} ; \\
& m_{I}^{f 2}=k(k+1+p) \bar{e}^{2}, \\
& m_{I}^{s 2}=k(k-1-p) \bar{e}^{2}, \\
& m_{I}^{t^{2}}=(k+1+p)(k+2+2 p) \bar{e}^{2} \text {; } \\
& G_{I_{1} I_{2} I_{3}}^{f f f}=\left(\alpha+\frac{1}{2}(1+p)\right) a_{I_{1} I_{2} I_{3}}\left\langle\mathcal{T}_{I_{1}} \mathcal{T}_{I_{2}} \mathcal{T}_{I_{3}}\right\rangle \bar{e}^{-p}, \\
& G_{I_{1} I_{2} I_{3}}^{f f s}=4(D-2) \frac{\alpha_{1} \alpha_{2}\left(\alpha_{3}+\frac{1}{2}(1+p)\right)\left(\alpha+\frac{1}{2}(1+p)\right)}{k_{3}+\gamma_{s}} a_{I_{1} I_{2} I_{3}}\left\langle\mathcal{T}_{I_{1}} \mathcal{T}_{I_{2}} \mathcal{C}_{I_{3}}\right\rangle \bar{e}^{-p}, \\
& G_{I_{1} I_{2} I_{3}}^{f f t}=4(D-2) \frac{\left(\alpha_{1}+\frac{1}{2}(1+p)\right)\left(\alpha_{2}+\frac{1}{2}(1+p)\right) \alpha_{3}(\alpha+1+p)}{k_{3}+\gamma_{t}} a_{I_{1} I_{2} I_{3}}\left\langle\mathcal{T}_{I_{1}} \mathcal{T}_{I_{2}} \mathcal{C}_{I_{3}}\right\rangle \bar{e}^{-p}, \\
& G_{I_{1} I_{2} I_{3}}^{f s s}=8 \nu \frac{\alpha_{1}\left(\alpha_{1}-\frac{1}{2}(1+p)\right) \alpha\left(\alpha+\frac{1}{2}(1+p)\right)}{\left(k_{2}+\gamma_{s}\right)\left(k_{3}+\gamma_{s}\right)} \\
& \times\left\{\left(\alpha_{1}-1\right)\left(\alpha+\frac{1+p}{D-3-p}\right)+\frac{\theta}{\nu} F^{f s s}\right\} a_{I_{1} I_{2} I_{3}}\left\langle\mathcal{T}_{I_{1}} \mathcal{C}_{I_{2}} \mathcal{C}_{I_{3}}\right\rangle \bar{e}^{-p}, \\
& G_{I_{1} I_{2} I_{3}}^{f t t}=8 \nu \frac{\alpha_{1}\left(\alpha_{1}+1+p\right)\left(\alpha+\frac{1}{2}(1+p)\right)\left(\alpha+\frac{3}{2}(1+p)\right)}{\left(k_{2}+\gamma_{t}\right)\left(k_{3}+\gamma_{t}\right)} \\
& \times\left\{\left(\alpha_{1}+\frac{(1+p)(D-4-p)}{D-3-p}\right)(\alpha+p+2)+\frac{\theta}{\nu} F^{f t t}\right\} a_{I_{1} I_{2} I_{3}}\left\langle\mathcal{T}_{I_{1}} \mathcal{C}_{I_{2}} \mathcal{C}_{I_{3}}\right\rangle \bar{e}^{-p}, \\
& G_{I_{1} I_{2} I_{3}}^{f s t}=8 \nu \frac{\alpha_{1}\left(\alpha_{2}+1+p\right)\left(\alpha_{3}-\frac{1}{2}(1+p)\right)\left(\alpha+\frac{1}{2}(1+p)\right)}{\left(k_{2}+\gamma_{s}\right)\left(k_{3}+\gamma_{t}\right)} \\
& \times\left\{\left(\alpha_{2}+\frac{(1+p)(D-4-p)}{D-3-p}\right)\left(\alpha_{3}-1\right)+\frac{\theta}{\nu} F^{f s t}\right\} a_{I_{1} I_{2} I_{3}}\left\langle\mathcal{T}_{I_{1}} \mathcal{C}_{I_{2}} \mathcal{C}_{I_{3}}\right\rangle \bar{e}^{-p}, \\
& G_{I_{1} I_{2} I_{3}}^{s s s}=32(D-2) \nu \frac{\alpha_{1} \alpha_{2} \alpha_{3}\left(\alpha-\frac{1}{2}(1+p)\right)\left(\alpha+\frac{1}{2}(1+p)\right)}{\left(k_{1}+\gamma_{s}\right)\left(k_{2}+\gamma_{s}\right)\left(k_{3}+\gamma_{s}\right)}
\end{aligned}
$$




$$
\begin{array}{rl} 
& \times\left\{(\alpha-1)\left(\alpha+\frac{1+p}{D-3-p}\right)\left(\alpha+\frac{(1+p)(-D+4+2 p)}{2(D-2)}\right)+\frac{\theta}{\nu} F^{s s s}\right\} \\
& \times a_{I_{1} I_{2} I_{3}}\left\langle\mathcal{C}_{I_{1}} \mathcal{C}_{I_{2}} \mathcal{C}_{I_{3}}\right\rangle \bar{e}^{-p}, \\
G_{I_{1} I_{2} I_{3}}^{s t}= & 32(D-2) \nu \frac{\left(\alpha_{1}+\frac{1}{2}(1+p)\right)\left(\alpha_{2}+\frac{1}{2}(1+p)\right) \alpha_{3}\left(\alpha_{3}-1-p\right)\left(\alpha+\frac{1}{2}(1+p)\right)}{\left(k_{1}+\gamma_{s}\right)\left(k_{2}+\gamma_{s}\right)\left(k_{3}+\gamma_{t}\right)} \\
& \times\left\{\left(\alpha_{3}-1\right)\left(\alpha_{3}+\frac{(1+p)(-D+3+p)}{D-2}\right)\left(\alpha_{3}+\frac{(1+p)(-D+4+p)}{D-3-p}\right)-\frac{\theta}{\nu} F^{s s t}\right\} \\
& \times a_{I_{1} I_{2} I_{3}}\left\langle\mathcal{C}_{I_{1}} \mathcal{C}_{I_{2}} \mathcal{C}_{I_{3}}\right\rangle \bar{e}^{-p}, \quad(2.4 h) \\
G_{I_{1} I_{2} I_{3}}^{t t s} & \left.32(D-2) \nu \frac{\alpha_{1} \alpha_{2}\left(\alpha_{3}+\frac{1}{2}(1+p)\right)\left(\alpha_{3}+\frac{3}{2}(1+p)\right)(\alpha+1+p)}{\left(k_{1}+\gamma_{t}\right)\left(k_{2}+\gamma_{t}\right)\left(k_{3}+\gamma_{s}\right)}\right) \quad(2.4 i) \\
& \times\left\{\left(\alpha_{3}+2+p\right)\left(\alpha_{3}+\frac{(1+p)(3 D-8-2 p)}{2(D-2)}\right)\left(\alpha_{3}+\frac{(1+p)(D-4-p)}{D-3-p} F^{t t s}\right\}\right. \\
& \left.\times a_{I_{1} I_{2} I_{3}}\left\langle\mathcal{C}_{I_{1}} \mathcal{C}_{I_{2}} \mathcal{C}_{I_{3}}\right\rangle \bar{e}^{-p}, \quad{ }^{t}\right) \\
G_{I_{1} I_{2} I_{3}}^{t t t} & 32(D-2) \nu \frac{\left(\alpha_{1}+\frac{1}{2}(1+p)\right)\left(\alpha_{2}+\frac{1}{2}(1+p)\right)\left(\alpha_{3}+\frac{1}{2}(1+p)\right)(\alpha+1+p)(\alpha+2+2 p)}{\left(k_{1}+\gamma_{t}\right)\left(k_{2}+\gamma_{t}\right)\left(k_{3}+\gamma_{t}\right)} \\
& \times\left\{(\alpha+2+p)\left(\alpha+\frac{(1+p)(2 D-8-2 p)}{D-3-p}\right)\left(\alpha+\frac{(1+p)(4 D-9-p)}{2(D-2)}\right)+\frac{\theta}{\nu} F^{t t t}\right\} \\
& \times a_{I_{1} I_{2} I_{3}}\left\langle\mathcal{C}_{I_{1}} \mathcal{C}_{I_{2}} \mathcal{C}_{I_{3}}\right\rangle \bar{e}^{-p}, \quad(2.4 j)
\end{array}
$$

where we have defined for notational convenience

$$
\begin{aligned}
\nu & =(D-2)(1+p)(D-3-p) \\
\gamma_{s} & =\frac{1+p}{D-3-p}, \quad \gamma_{t}=\frac{(1+p)(D-4-p)}{D-3-p} \\
\theta & =(1+p)(D-3-p)-2(D-2)
\end{aligned}
$$

$\bar{e}^{2}$ is defined in eq. (1.5b), while $\alpha_{1}, \alpha_{2}, \alpha_{3}, \alpha, z_{I}, a_{I_{1} I_{2} I_{3}}$ and the contractions $\langle\mathcal{T} \mathcal{T} \mathcal{T}\rangle$, $\langle\mathcal{T} \mathcal{T C}\rangle,\langle\mathcal{T C C}\rangle,\langle\mathcal{C C C}\rangle$ are defined in appendix A1. Note that the auxiliary functions $F^{f s s}, F^{f t t}, \ldots$ appearing in the couplings are always multiplied by $\theta$ and do not contribute to the relevant cases of $\mathrm{AdS}_{5,7,4} \times \mathrm{S}_{5,4,7}$ where $\theta$ vanishes. The explicit expressions for such functions, which may be needed in future applications, are listed in appendix A2.

Writing above

$$
\begin{gathered}
A_{I}=\bar{A}_{I} z_{I} \bar{e}^{-2-p} \\
m_{I}^{2}=\bar{m}_{I}^{2} \bar{e}^{2} \\
G_{I_{1} I_{2} I_{3}}=\bar{G}_{I_{1} I_{2} I_{3}} a_{I_{1} I_{2} I_{3}}\left\langle\mathcal{Y}_{I_{1}} \mathcal{Y}_{I_{2}} \mathcal{Y}_{I_{3}}\right\rangle \bar{e}^{-p},
\end{gathered}
$$

where the $\left\langle\mathcal{Y}_{I_{1}} \mathcal{Y}_{I_{2}} \mathcal{Y}_{I_{3}}\right\rangle$ denotes the appropriate tensor/scalar harmonic contractions, we get the following expressions. 
$\underline{\mathrm{AdS}_{7} \times \mathrm{S}_{4}}$

$$
\begin{aligned}
& \bar{A}_{I}^{f}=\frac{1}{2}, \\
& \bar{A}_{I}^{s}=\frac{2^{2} 3^{4} k(k-1)(2 k+3)}{k+\frac{1}{2}}, \\
& \bar{A}_{I}^{t}=\frac{2^{2} 3^{4}(k+3)(k+4)(2 k+3)}{k+\frac{5}{2}} ; \\
& \bar{m}_{I}^{f 2}=k(k+3) \text {, } \\
& \bar{m}_{I}^{s 2}=k(k-3) \text {, } \\
& \bar{m}_{I}^{t 2}=(k+3)(k+6) \text {; } \\
& \bar{G}_{I_{1} I_{2} I_{3}}^{f f f}=\alpha+\frac{3}{2}, \\
& \bar{G}_{I_{1} I_{2} I_{3}}^{f f s}=\frac{2^{2} 3^{2} \alpha_{1} \alpha_{2}\left(\alpha_{3}+\frac{3}{2}\right)\left(\alpha+\frac{3}{2}\right)}{k_{3}+\frac{1}{2}}, \\
& \bar{G}_{I_{1} I_{2} I_{3}}^{f f t}=\frac{2^{2} 3^{2}\left(\alpha_{1}+\frac{3}{2}\right)\left(\alpha_{2}+\frac{3}{2}\right) \alpha_{3}(\alpha+3)}{k_{3}+\frac{5}{2}}, \\
& \bar{G}_{I_{1} I_{2} I_{3}}^{f s s}=\frac{2^{4} 3^{4}\left(\alpha_{1}-\frac{3}{2}\right)\left(\alpha_{1}-1\right) \alpha_{1} \alpha\left(\alpha+\frac{1}{2}\right)\left(\alpha+\frac{3}{2}\right)}{\left(k_{2}+\frac{1}{2}\right)\left(k_{3}+\frac{1}{2}\right)}, \\
& \bar{G}_{I_{1} I_{2} I_{3}}^{f t t}=\frac{2^{4} 3^{4} \alpha_{1}\left(\alpha_{1}+\frac{5}{2}\right)\left(\alpha_{1}+3\right)\left(\alpha+\frac{3}{2}\right)(\alpha+4)\left(\alpha+\frac{9}{2}\right)}{\left(k_{2}+\frac{5}{2}\right)\left(k_{3}+\frac{5}{2}\right)}, \\
& \bar{G}_{I_{1} I_{2} I_{3}}^{f s t}=\frac{2^{4} 3^{4} \alpha_{1}\left(\alpha_{2}+\frac{5}{2}\right)\left(\alpha_{2}+3\right)\left(\alpha_{3}-1\right)\left(\alpha_{3}-\frac{3}{2}\right)\left(\alpha+\frac{3}{2}\right)}{\left(k_{2}+\frac{1}{2}\right)\left(k_{3}+\frac{5}{2}\right)}, \\
& \bar{G}_{I_{1} I_{2} I_{3}}^{s s s}=\frac{2^{6} 3^{6} \alpha_{1} \alpha_{2} \alpha_{3}(\alpha-1)\left(\alpha^{2}-\frac{1}{4}\right)\left(\alpha^{2}-\frac{9}{4}\right)}{\left(k_{1}+\frac{1}{2}\right)\left(k_{2}+\frac{1}{2}\right)\left(k_{3}+\frac{1}{2}\right)}, \\
& \bar{G}_{I_{1} I_{2} I_{3}}^{s s t}=\frac{2^{6} 3^{6}\left(\alpha_{1}+\frac{3}{2}\right)\left(\alpha_{2}+\frac{3}{2}\right) \alpha_{3}\left(\alpha_{3}-1\right)\left(\alpha_{3}-2\right)\left(\alpha_{3}-\frac{5}{2}\right)\left(\alpha_{3}-3\right)\left(\alpha+\frac{3}{2}\right)}{\left(k_{1}+\frac{1}{2}\right)\left(k_{2}+\frac{1}{2}\right)\left(k_{3}+\frac{5}{2}\right)}, \\
& \bar{G}_{I_{1} I_{2} I_{3}}^{t t s}=\frac{2^{6} 3^{6} \alpha_{1} \alpha_{2}\left(\alpha_{3}+\frac{3}{2}\right)\left(\alpha_{3}+\frac{5}{2}\right)\left(\alpha_{3}+\frac{7}{2}\right)\left(\alpha_{3}+4\right)\left(\alpha_{3}+\frac{9}{2}\right)(\alpha+3)}{\left(k_{1}+\frac{5}{2}\right)\left(k_{2}+\frac{5}{2}\right)\left(k_{3}+\frac{1}{2}\right)}, \\
& \bar{G}_{I_{1} I_{2} I_{3}}^{t t t}=\frac{2^{6} 3^{6}\left(\alpha_{1}+\frac{3}{2}\right)\left(\alpha_{2}+\frac{3}{2}\right)\left(\alpha_{3}+\frac{3}{2}\right)(\alpha+3)(\alpha+4)(\alpha+5)\left(\alpha+\frac{11}{2}\right)(\alpha+6)}{\left(k_{1}+\frac{5}{2}\right)\left(k_{2}+\frac{5}{2}\right)\left(k_{3}+\frac{5}{2}\right)} .
\end{aligned}
$$


$\underline{\mathrm{AdS}_{4} \times \mathrm{S}_{7}}$

$$
\begin{aligned}
& \bar{A}_{I}^{f}=\frac{1}{2}, \\
& \bar{A}_{I}^{s}=\frac{2^{2} 3^{4} k(k-1)(2 k+6)}{k+2}, \\
& \bar{A}_{I}^{t}=\frac{2^{2} 3^{4}(k+6)(k+7)(2 k+6)}{k+4} ; \\
& \bar{m}_{I}^{f 2}=k(k+6), \\
& \bar{m}_{I}^{s}{ }^{2}=k(k-6) \text {, } \\
& \bar{m}_{I}^{t 2}=(k+6)(k+12) \text {; } \\
& \bar{G}_{I_{1} I_{2} I_{3}}^{f f f}=\alpha+3 \text {, } \\
& \bar{G}_{I_{1} I_{2} I_{3}}^{f f s}=\frac{2^{2} 3^{2} \alpha_{1} \alpha_{2}\left(\alpha_{3}+3\right)(\alpha+3)}{k_{3}+2}, \\
& \bar{G}_{I_{1} I_{2} I_{3}}^{f f t}=\frac{2^{2} 3^{2}\left(\alpha_{1}+3\right)\left(\alpha_{2}+3\right) \alpha_{3}(\alpha+6)}{k_{3}+4}, \\
& \bar{G}_{I_{1} I_{2} I_{3}}^{f s s}=\frac{2^{4} 3^{4}\left(\alpha_{1}-3\right)\left(\alpha_{1}-1\right) \alpha_{1} \alpha(\alpha+2)(\alpha+3)}{\left(k_{2}+2\right)\left(k_{3}+2\right)}, \\
& \bar{G}_{I_{1} I_{2} I_{3}}^{f t t}=\frac{2^{4} 3^{4} \alpha_{1}\left(\alpha_{1}+4\right)\left(\alpha_{1}+6\right)(\alpha+3)(\alpha+7)(\alpha+9)}{\left(k_{2}+4\right)\left(k_{3}+4\right)}, \\
& \bar{G}_{I_{1} I_{2} I_{3}}^{f s t}=\frac{2^{4} 3^{4} \alpha_{1}\left(\alpha_{2}+4\right)\left(\alpha_{2}+6\right)\left(\alpha_{3}-3\right)\left(\alpha_{3}-1\right)(\alpha+3)}{\left(k_{2}+2\right)\left(k_{3}+4\right)}, \\
& \bar{G}_{I_{1} I_{2} I_{3}}^{s s s}=\frac{2^{6} 3^{6} \alpha_{1} \alpha_{2} \alpha_{3}(\alpha+2)\left(\alpha^{2}-1\right)\left(\alpha^{2}-9\right)}{\left(k_{1}+2\right)\left(k_{2}+2\right)\left(k_{3}+2\right)}, \\
& \bar{G}_{I_{1} I_{2} I_{3}}^{s s t}=\frac{2^{6} 3^{6}\left(\alpha_{1}+3\right)\left(\alpha_{2}+3\right) \alpha_{3}\left(\alpha_{3}-1\right)\left(\alpha_{3}-2\right)\left(\alpha_{3}-4\right)\left(\alpha_{3}-6\right)(\alpha+3)}{\left(k_{1}+2\right)\left(k_{2}+2\right)\left(k_{3}+4\right)}, \\
& \bar{G}_{I_{1} I_{2} I_{3}}^{t t s}=\frac{2^{6} 3^{6} \alpha_{1} \alpha_{2}\left(\alpha_{3}+3\right)\left(\alpha_{3}+4\right)\left(\alpha_{3}+5\right)\left(\alpha_{3}+7\right)\left(\alpha_{3}+9\right)(\alpha+6)}{\left(k_{1}+4\right)\left(k_{2}+4\right)\left(k_{3}+2\right)}, \\
& \bar{G}_{I_{1} I_{2} I_{3}}^{t t t}=\frac{2^{6} 3^{6}\left(\alpha_{1}+3\right)\left(\alpha_{2}+3\right)\left(\alpha_{3}+3\right)(\alpha+6)(\alpha+7)(\alpha+8)(\alpha+10)(\alpha+12)}{\left(k_{1}+4\right)\left(k_{2}+4\right)\left(k_{3}+4\right)} \text {. }
\end{aligned}
$$


$\underline{\mathrm{AdS}_{5} \times \mathrm{S}_{5}}$

$$
\begin{aligned}
& \bar{A}_{I}^{f}=\frac{1}{2}, \\
& \bar{A}_{I}^{s}=\frac{2^{8} k(k-1)(2 k+4)}{k+1}, \\
& \bar{A}_{I}^{t}=\frac{2^{8}(k+4)(k+5)(2 k+4)}{k+3} ; \\
& \bar{m}_{I}^{f 2}=k(k+4), \\
& \bar{m}_{I}^{s 2}=k(k-4) \text {, } \\
& \bar{m}_{I}^{t 2}=(k+4)(k+8) \text {; } \\
& \bar{G}_{I_{1} I_{2} I_{3}}^{f f s}=\frac{2^{5} \alpha_{1} \alpha_{2}\left(\alpha_{3}+2\right)(\alpha+2)}{k_{3}+1}, \\
& \bar{G}_{I_{1} I_{2} I_{3}}^{f f t}=\frac{2^{5}\left(\alpha_{1}+2\right)\left(\alpha_{2}+2\right) \alpha_{3}(\alpha+4)}{k_{3}+3}, \\
& \bar{G}_{I_{1} I_{2} I_{3}}^{f s s}=\frac{2^{10}\left(\alpha_{1}-2\right)\left(\alpha_{1}-1\right) \alpha_{1} \alpha(\alpha+1)(\alpha+2)}{\left(k_{2}+1\right)\left(k_{3}+1\right)}, \\
& \bar{G}_{I_{1} I_{2} I_{3}}^{f t t}=\frac{2^{10} \alpha_{1}\left(\alpha_{1}+3\right)\left(\alpha_{1}+4\right)(\alpha+2)(\alpha+5)(\alpha+6)}{\left(k_{2}+3\right)\left(k_{3}+3\right)} \text {, } \\
& \bar{G}_{I_{1} I_{2} I_{3}}^{f s t}=\frac{2^{10} \alpha_{1}\left(\alpha_{2}+3\right)\left(\alpha_{2}+4\right)\left(\alpha_{3}-2\right)\left(\alpha_{3}-1\right)(\alpha+2)}{\left(k_{2}+1\right)\left(k_{3}+3\right)}, \\
& \bar{G}_{I_{1} I_{2} I_{3}}^{s s s}=\frac{2^{15} \alpha_{1} \alpha_{2} \alpha_{3} \alpha\left(\alpha^{2}-1\right)\left(\alpha^{2}-4\right)}{\left(k_{1}+1\right)\left(k_{2}+1\right)\left(k_{3}+1\right)}, \\
& \bar{G}_{I_{1} I_{2} I_{3}}^{s s t}=\frac{2^{15}\left(\alpha_{1}+2\right)\left(\alpha_{2}+2\right) \alpha_{3}\left(\alpha_{3}-1\right)\left(\alpha_{3}-2\right)\left(\alpha_{3}-3\right)\left(\alpha_{3}-4\right)(\alpha+2)}{\left(k_{1}+1\right)\left(k_{2}+1\right)\left(k_{3}+3\right)} \text {, } \\
& \bar{G}_{I_{1} I_{2} I_{3}}^{t t s}=\frac{2^{15} \alpha_{1} \alpha_{2}\left(\alpha_{3}+2\right)\left(\alpha_{3}+3\right)\left(\alpha_{3}+4\right)\left(\alpha_{3}+5\right)\left(\alpha_{3}+6\right)(\alpha+4)}{\left(k_{1}+3\right)\left(k_{2}+3\right)\left(k_{3}+1\right)}, \\
& \bar{G}_{I_{1} I_{2} I_{3}}^{t t t}=\frac{2^{15}\left(\alpha_{1}+2\right)\left(\alpha_{2}+2\right)\left(\alpha_{3}+2\right)(\alpha+4)(\alpha+5)(\alpha+6)(\alpha+7)(\alpha+8)}{\left(k_{1}+3\right)\left(k_{2}+3\right)\left(k_{3}+3\right)} .
\end{aligned}
$$




\section{Computation of the 3 point functions of the universal scalar sector}

We are now ready to compute two and three point functions in the SCFTs using the $\mathrm{AdS}_{d+1} / \mathrm{CFT}_{d}$ correspondence. The general formulas derived in [29,30] work with AdS radius set to 1 . Assume that the AdS scalar fields $\phi_{i}$ correspond to the CFT local field $\mathcal{O}_{i}$. The mass $m_{i}$ of $\psi_{i}$ and the conformal dimension $\Delta_{i}$ of $\mathcal{O}_{i}$ are related as

$$
\Delta_{i}=\frac{1}{2}\left[d+\left(d^{2}+4 m_{i}^{2}\right)^{\frac{1}{2}}\right]
$$

Then

$$
\left\langle\mathcal{O}_{i}(x) \mathcal{O}_{j}(y)\right\rangle=\frac{A_{i}}{4 \kappa^{2}} \frac{2}{\pi^{\frac{d}{2}}} \frac{\Delta_{i}-\frac{d}{2}}{\Delta_{i}} \frac{\Gamma\left(\Delta_{i}+1\right)}{\Gamma\left(\Delta_{i}-\frac{d}{2}\right)} \frac{\left(w_{i}\right)^{2} \delta_{i j}}{|x-y|^{2 \Delta_{i}}},
$$

where $\frac{A_{i}}{4 \kappa^{2}}$ is the coefficient of the canonically normalized kinetic term of the bulk field $\psi_{i}$ as in eq. (2.1) and

$$
\left\langle\mathcal{O}_{i}(x) \mathcal{O}_{j}(y) \mathcal{O}_{k}(z)\right\rangle=\frac{R_{i j k}}{|x-y|^{\Delta_{i}+\Delta_{j}-\Delta_{k}}|y-z|^{\Delta_{j}+\Delta_{k}-\Delta_{i}}|z-x|^{\Delta_{k}+\Delta_{i}-\Delta_{j}}},
$$

with

$$
\begin{aligned}
R_{i j k}= & \frac{G_{i j k}}{4 \kappa^{2}} \frac{1}{2 \pi^{d}} \frac{\Gamma\left(\frac{1}{2}\left(\Delta_{i}+\Delta_{j}-\Delta_{k}\right)\right) \Gamma\left(\frac{1}{2}\left(\Delta_{j}+\Delta_{k}-\Delta_{i}\right)\right) \Gamma\left(\frac{1}{2}\left(\Delta_{k}+\Delta_{i}-\Delta_{j}\right)\right)}{\Gamma\left(\Delta_{i}-\frac{d}{2}\right) \Gamma\left(\Delta_{j}-\frac{d}{2}\right) \Gamma\left(\Delta_{k}-\frac{d}{2}\right)} \\
& \Gamma\left(\frac{1}{2}\left(\Delta_{i}+\Delta_{j}+\Delta_{k}-d\right)\right) w_{i} w_{j} w_{k},
\end{aligned}
$$

where $\frac{G_{i j k}}{4 \kappa^{2}}$ is the cubic coupling constant of $\psi_{i}, \psi_{j}, \psi_{k}$ as in eq. (2.1). The factors $w_{i}$ parameterize unknown proportionality constants which relate the fields $\psi_{i}$ to the sources of the operators $\mathcal{O}_{i}$, namely the generating functional of correlators reads as $\left\langle e \int w_{i} \psi_{i} \mathcal{O}_{i}\right\rangle_{\mathrm{SCFT}}$. For the present purposes we follow ref. [13] and fix them to normalize the two point functions as

$$
\left\langle\mathcal{O}_{i}(x) \mathcal{O}_{j}(y)\right\rangle=\frac{\delta_{i j}}{|x-y|^{2 \Delta_{i}}}
$$

With this canonical normalization the three point functions are readily computed.

In our case, $d=D-3-p$. Imposing that the AdS radius is 1 fixes the value of $\bar{e}$ to be

$$
\bar{e}=\frac{d}{1+p} .
$$

We denote by $\mathcal{O}_{I}^{f}, \mathcal{O}_{I}^{s}, \mathcal{O}_{I}^{t}$ the $\mathrm{CFT}_{d}$ operators corresponding to the $\mathrm{AdS}_{d+1}$ scalars $f_{I}$, $s_{I}, t_{I}$ in the $\mathrm{AdS}_{d+1} / \mathrm{CFT}_{d}$ duality. Their dimensions are given by

$$
\begin{aligned}
& \Delta_{I}^{f}=\frac{d(k+1+p)}{1+p} \\
& \Delta_{I}^{s}=\frac{d k}{1+p} \\
& \Delta_{I}^{t}=\frac{d(k+2+2 p)}{1+p} .
\end{aligned}
$$


Separating out the group theory factors in the $R_{i j k}$ coefficients by defining

$$
R_{i j k}=\bar{R}_{i j k}\left\langle\mathcal{Y}_{I_{1}} \mathcal{Y}_{I_{2}} \mathcal{Y}_{I_{3}}\right\rangle
$$

as in eq. (2.8), we find the following expressions.

\section{$\underline{\mathrm{AdS}_{7} \times \mathrm{S}_{4}}$}

In this case one has $\frac{1}{4 \kappa^{2}}=\frac{2 N^{3}}{\pi^{5}}$ and $\bar{e}=2$. One has

$$
\begin{aligned}
\Delta_{I}^{f} & =2 k+6, \\
\Delta_{I}^{s} & =2 k, \\
\Delta_{I}^{t} & =2 k+12 .
\end{aligned}
$$

Set

$$
\begin{aligned}
\phi(k) & =4\left[\frac{(2 k+1) !}{(2 k+2) !(2 k+5) !}\right]^{\frac{1}{2}}, \\
\sigma(k) & =[(2 k-2) !]^{-\frac{1}{2}} \\
\tau(k) & =16\left[\frac{(2 k+1) !(2 k+4) !(2 k+7) !}{(2 k+6) !(2 k+8) !(2 k+9) !(2 k+11) !}\right]^{\frac{1}{2}} .
\end{aligned}
$$

Then,

$$
\begin{aligned}
\bar{R}_{I_{1} I_{2} I_{3}}^{f f f} & =\frac{2}{4(\pi N)^{\frac{3}{2}}} \phi\left(k_{1}\right) \phi\left(k_{2}\right) \phi\left(k_{3}\right) 2^{2 \alpha} \frac{\Gamma(2 \alpha+4) \Gamma(2 \alpha+6)}{\Gamma(2 \alpha+3) \Gamma(2 \alpha+5)} \Gamma(\alpha+3) \\
& \times \frac{\Gamma\left(2 \alpha_{1}+3\right) \Gamma\left(2 \alpha_{2}+3\right) \Gamma\left(2 \alpha_{3}+3\right)}{\Gamma\left(2 \alpha_{1}+2\right) \Gamma\left(2 \alpha_{2}+2\right) \Gamma\left(2 \alpha_{3}+2\right)} \Gamma\left(\alpha_{1}+\frac{3}{2}\right) \Gamma\left(\alpha_{2}+\frac{3}{2}\right) \Gamma\left(\alpha_{3}+\frac{3}{2}\right) \\
\bar{R}_{I_{1} I_{2} I_{3}}^{f f s} & =\frac{1}{4(\pi N)^{\frac{3}{2}}} \phi\left(k_{1}\right) \phi\left(k_{2}\right) \sigma\left(k_{3}\right) 2^{2 \alpha} \Gamma(\alpha+2) \\
& \times \frac{\Gamma\left(2 \alpha_{3}+6\right)}{\Gamma\left(2 \alpha_{3}+2\right)} \Gamma\left(\alpha_{1}+\frac{1}{2}\right) \Gamma\left(\alpha_{2}+\frac{1}{2}\right) \Gamma\left(\alpha_{3}+\frac{5}{2}\right), \\
\bar{R}_{I_{1} I_{2} I_{3}}^{f f t} & =\frac{1}{4(\pi N)^{\frac{3}{2}}} \phi\left(k_{1}\right) \phi\left(k_{2}\right) \tau\left(k_{3}\right) 2^{2 \alpha} \frac{\Gamma(2 \alpha+9)}{\Gamma(2 \alpha+5)} \Gamma(\alpha+4) \\
& \times \frac{\Gamma\left(2 \alpha_{1}+6\right) \Gamma\left(2 \alpha_{2}+6\right)}{\Gamma\left(2 \alpha_{1}+2\right) \Gamma\left(2 \alpha_{2}+2\right)} \Gamma\left(\alpha_{1}+\frac{5}{2}\right) \Gamma\left(\alpha_{2}+\frac{5}{2}\right) \Gamma\left(\alpha_{3}+\frac{1}{2}\right), \\
\bar{R}_{I_{1} I_{2} I_{3}}^{f s s} & =\frac{1}{4(\pi N)^{\frac{3}{2}}} \phi\left(k_{1}\right) \sigma\left(k_{2}\right) \sigma\left(k_{3}\right) 2^{2 \alpha} \Gamma(\alpha+1) \\
& \times \frac{\Gamma\left(2 \alpha_{2}+3\right) \Gamma\left(2 \alpha_{3}+3\right)}{\Gamma\left(2 \alpha_{2}+2\right) \Gamma\left(2 \alpha_{3}+2\right)} \Gamma\left(\alpha_{1}-\frac{1}{2}\right) \Gamma\left(\alpha_{2}+\frac{3}{2}\right) \Gamma\left(\alpha_{3}+\frac{3}{2}\right),
\end{aligned}
$$




$$
\begin{aligned}
& \bar{R}_{I_{1} I_{2} I_{3}}^{f s t}=\frac{1}{4(\pi N)^{\frac{3}{2}}} \phi\left(k_{1}\right) \sigma\left(k_{2}\right) \tau\left(k_{3}\right) 2^{2 \alpha} \frac{\Gamma(2 \alpha+4) \Gamma(2 \alpha+6)}{\Gamma(2 \alpha+3) \Gamma(2 \alpha+5)} \Gamma(\alpha+3) \\
& \times \frac{\Gamma\left(2 \alpha_{1}+1\right) \Gamma\left(2 \alpha_{1}+3\right)}{\Gamma\left(2 \alpha_{1}\right) \Gamma\left(2 \alpha_{1}+2\right)} \frac{\Gamma\left(2 \alpha_{2}+3\right) \Gamma\left(2 \alpha_{2}+7\right) \Gamma\left(2 \alpha_{2}+9\right)}{\Gamma\left(2 \alpha_{2}+2\right) \Gamma\left(2 \alpha_{2}+4\right) \Gamma\left(2 \alpha_{2}+6\right)} \frac{\Gamma\left(2 \alpha_{3}\right)}{\Gamma\left(2 \alpha_{3}+1\right)} \\
& \times \Gamma\left(\alpha_{1}+\frac{3}{2}\right) \Gamma\left(\alpha_{2}+\frac{7}{2}\right) \Gamma\left(\alpha_{3}-\frac{1}{2}\right), \\
& \bar{R}_{I_{1} I_{2} I_{3}}^{f t t}=\frac{1}{4(\pi N)^{\frac{3}{2}}} \phi\left(k_{1}\right) \tau\left(k_{2}\right) \tau\left(k_{3}\right) \\
& \times 2^{2 \alpha} \frac{\Gamma(2 \alpha+4) \Gamma(2 \alpha+6) \Gamma(2 \alpha+10) \Gamma(2 \alpha+12)}{\Gamma(2 \alpha+3) \Gamma(2 \alpha+5) \Gamma(2 \alpha+7) \Gamma(2 \alpha+9)} \Gamma(\alpha+5) \\
& \times \frac{\Gamma\left(2 \alpha_{1}+1\right) \Gamma\left(2 \alpha_{1}+3\right) \Gamma\left(2 \alpha_{1}+7\right) \Gamma\left(2 \alpha_{1}+9\right)}{\Gamma\left(2 \alpha_{1}\right) \Gamma\left(2 \alpha_{1}+2\right) \Gamma\left(2 \alpha_{1}+4\right) \Gamma\left(2 \alpha_{1}+6\right)} \frac{\Gamma\left(2 \alpha_{2}+3\right)}{\Gamma\left(2 \alpha_{2}+2\right)} \frac{\Gamma\left(2 \alpha_{3}+3\right)}{\Gamma\left(2 \alpha_{3}+2\right)} \\
& \times \Gamma\left(\alpha_{1}+\frac{7}{2}\right) \Gamma\left(\alpha_{2}+\frac{3}{2}\right) \Gamma\left(\alpha_{3}+\frac{3}{2}\right), \\
& \bar{R}_{I_{1} I_{2} I_{3}}^{s s s}=\frac{1}{4(\pi N)^{\frac{3}{2}}} \sigma\left(k_{1}\right) \sigma\left(k_{2}\right) \sigma\left(k_{3}\right) 2^{2 \alpha} \Gamma(\alpha) \Gamma\left(\alpha_{1}+\frac{1}{2}\right) \Gamma\left(\alpha_{2}+\frac{1}{2}\right) \Gamma\left(\alpha_{3}+\frac{1}{2}\right), \\
& \bar{R}_{I_{1} I_{2} I_{3}}^{s s t}=\frac{1}{4(\pi N)^{\frac{3}{2}}} \sigma\left(k_{1}\right) \sigma\left(k_{2}\right) \tau\left(k_{3}\right) 2^{2 \alpha} \Gamma(\alpha+2) \\
& \times \frac{\Gamma\left(2 \alpha_{1}+6\right) \Gamma\left(2 \alpha_{2}+6\right)}{\Gamma\left(2 \alpha_{1}+2\right) \Gamma\left(2 \alpha_{2}+2\right)} \Gamma\left(\alpha_{1}+\frac{5}{2}\right) \Gamma\left(\alpha_{2}+\frac{5}{2}\right) \Gamma\left(\alpha_{3}-\frac{3}{2}\right), \\
& \bar{R}_{I_{1} I_{2} I_{3}}^{t t s}=\frac{1}{4(\pi N)^{\frac{3}{2}}} \tau\left(k_{1}\right) \tau\left(k_{2}\right) \sigma\left(k_{3}\right) 2^{2 \alpha} \frac{\Gamma(2 \alpha+9)}{\Gamma(2 \alpha+5)} \Gamma(\alpha+4) \\
& \times \frac{\Gamma\left(2 \alpha_{3}+10\right) \Gamma\left(2 \alpha_{3}+12\right)}{\Gamma\left(2 \alpha_{3}+2\right) \Gamma\left(2 \alpha_{3}+8\right)} \Gamma\left(\alpha_{1}+\frac{1}{2}\right) \Gamma\left(\alpha_{2}+\frac{1}{2}\right) \Gamma\left(\alpha_{3}+\frac{9}{2}\right), \\
& \bar{R}_{I_{1} I_{2} I_{3}}^{t t t}=\frac{1}{4(\pi N)^{\frac{3}{2}}} \tau\left(k_{1}\right) \tau\left(k_{2}\right) \tau\left(k_{3}\right) 2^{2 \alpha} \frac{\Gamma(2 \alpha+13) \Gamma(2 \alpha+15)}{\Gamma(2 \alpha+5) \Gamma(2 \alpha+11)} \Gamma(\alpha+6) \\
& \times \frac{\Gamma\left(2 \alpha_{1}+6\right) \Gamma\left(2 \alpha_{2}+6\right) \Gamma\left(2 \alpha_{3}+6\right)}{\Gamma\left(2 \alpha_{1}+2\right) \Gamma\left(2 \alpha_{2}+2\right) \Gamma\left(2 \alpha_{3}+2\right)} \Gamma\left(\alpha_{1}+\frac{5}{2}\right) \Gamma\left(\alpha_{2}+\frac{5}{2}\right) \Gamma\left(\alpha_{3}+\frac{5}{2}\right) .
\end{aligned}
$$

$\underline{\mathrm{AdS}_{4} \times \mathrm{S}_{7}}$

In this case one has $\frac{1}{4 \kappa^{2}}=\frac{N^{\frac{3}{2}}}{2^{\frac{19}{2}} \pi^{5}}$ and $\bar{e}=\frac{1}{2}$. One has

$$
\begin{aligned}
\Delta_{I}^{f} & =\frac{1}{2} k+3, \\
\Delta_{I}^{s} & =\frac{1}{2} k, \\
\Delta_{I}^{t} & =\frac{1}{2} k+6 .
\end{aligned}
$$

Set

$$
\phi(k)=\frac{1}{2}\left[\frac{k !(k+3) !}{(k+4) !}\right]^{\frac{1}{2}}
$$




$$
\begin{aligned}
\sigma(k) & =[(k+1) !]^{\frac{1}{2}} \\
\tau(k) & =\frac{1}{4}\left[\frac{k !(k+2) !(k+3) !(k+5) !}{(k+4) !(k+7) !(k+10) !}\right]^{\frac{1}{2}}
\end{aligned}
$$

Then,

$$
\begin{aligned}
& \bar{R}_{I_{1} I_{2} I_{3}}^{f f f}=\frac{\pi}{2}\left(\frac{2}{N}\right)^{\frac{3}{4}} \phi\left(k_{1}\right) \phi\left(k_{2}\right) \phi\left(k_{3}\right) 2^{-\alpha} \frac{\Gamma(\alpha+5)}{\Gamma(\alpha+3)} \frac{1}{\Gamma\left(\frac{1}{2} \alpha+\frac{5}{2}\right)} \\
& \times \frac{\Gamma\left(\alpha_{1}+2\right) \Gamma\left(\alpha_{2}+2\right) \Gamma\left(\alpha_{3}+2\right)}{\Gamma\left(\alpha_{1}+1\right) \Gamma\left(\alpha_{2}+1\right) \Gamma\left(\alpha_{3}+1\right)} \frac{1}{\Gamma\left(\frac{1}{2} \alpha_{1}+1\right) \Gamma\left(\frac{1}{2} \alpha_{2}+1\right) \Gamma\left(\frac{1}{2} \alpha_{3}+1\right)} \\
& \bar{R}_{I_{1} I_{2} I_{3}}^{f f s}=\frac{\pi}{2}\left(\frac{2}{N}\right)^{\frac{3}{4}} \phi\left(k_{1}\right) \phi\left(k_{2}\right) \sigma\left(k_{3}\right) 2^{-\alpha} \frac{1}{\Gamma\left(\frac{1}{2} \alpha+2\right)} \\
& \times \frac{\Gamma\left(\alpha_{3}+5\right)}{\Gamma\left(\alpha_{3}+1\right)} \frac{1}{\Gamma\left(\frac{1}{2}\left(\alpha_{1}+1\right)\right) \Gamma\left(\frac{1}{2}\left(\alpha_{2}+1\right)\right) \Gamma\left(\frac{1}{2}\left(\alpha_{3}+3\right)\right)}, \\
& \bar{R}_{I_{1} I_{2} I_{3}}^{f f t}=\frac{\pi}{2}\left(\frac{2}{N}\right)^{\frac{3}{4}} \phi\left(k_{1}\right) \phi\left(k_{2}\right) \tau\left(k_{3}\right) 2^{-\alpha} \frac{\Gamma(\alpha+8)}{\Gamma(\alpha+4)} \frac{1}{\Gamma\left(\frac{1}{2} \alpha+3\right)} \\
& \times \frac{\Gamma\left(\alpha_{1}+5\right) \Gamma\left(\alpha_{2}+5\right)}{\Gamma\left(\alpha_{1}+1\right) \Gamma\left(\alpha_{2}+1\right)} \frac{1}{\Gamma\left(\frac{1}{2}\left(\alpha_{1}+3\right)\right) \Gamma\left(\frac{1}{2}\left(\alpha_{2}+3\right)\right) \Gamma\left(\frac{1}{2}\left(\alpha_{3}+1\right)\right)}, \\
& \bar{R}_{I_{1} I_{2} I_{3}}^{f s s}=\frac{\pi}{2}\left(\frac{2}{N}\right)^{\frac{3}{4}} \phi\left(k_{1}\right) \sigma\left(k_{2}\right) \sigma\left(k_{3}\right) 2^{-\alpha} \frac{1}{\Gamma\left(\frac{1}{2} \alpha+\frac{3}{2}\right)} \\
& \times \frac{\Gamma\left(\alpha_{2}+2\right) \Gamma\left(\alpha_{3}+2\right)}{\Gamma\left(\alpha_{2}+1\right) \Gamma\left(\alpha_{3}+1\right)} \frac{1}{\Gamma\left(\frac{1}{2} \alpha_{1}\right) \Gamma\left(\frac{1}{2} \alpha_{2}+1\right) \Gamma\left(\frac{1}{2} \alpha_{3}+1\right)}, \\
& \bar{R}_{I_{1} I_{2} I_{3}}^{f s t}=\frac{\pi}{2}\left(\frac{2}{N}\right)^{\frac{3}{4}} \phi\left(k_{1}\right) \sigma\left(k_{2}\right) \tau\left(k_{3}\right) 2^{-\alpha} \frac{\Gamma(\alpha+5)}{\Gamma(\alpha+3)} \frac{1}{\Gamma\left(\frac{1}{2} \alpha+\frac{5}{2}\right)} \\
& \times \frac{\Gamma\left(\alpha_{1}+2\right)}{\Gamma\left(\alpha_{1}\right)} \frac{\Gamma\left(\alpha_{2}+8\right)}{\Gamma\left(\alpha_{2}+1\right)} \frac{\Gamma\left(\alpha_{3}\right)}{\Gamma\left(\alpha_{3}+1\right)} \frac{1}{\Gamma\left(\frac{1}{2} \alpha_{1}+1\right) \Gamma\left(\frac{1}{2} \alpha_{2}+2\right) \Gamma\left(\frac{1}{2} \alpha_{3}\right)}, \\
& \bar{R}_{I_{1} I_{2} I_{3}}^{f t t}=\frac{\pi}{2}\left(\frac{2}{N}\right)^{\frac{3}{4}} \phi\left(k_{1}\right) \tau\left(k_{2}\right) \tau\left(k_{3}\right) 2^{-\alpha} \frac{\Gamma(\alpha+11)}{\Gamma(\alpha+3)} \frac{1}{\Gamma\left(\frac{1}{2} \alpha+\frac{7}{2}\right)} \\
& \times \frac{\Gamma\left(\alpha_{1}+8\right)}{\Gamma\left(\alpha_{1}\right)} \frac{\Gamma\left(\alpha_{2}+2\right)}{\Gamma\left(\alpha_{2}+1\right)} \frac{\Gamma\left(\alpha_{3}+2\right)}{\Gamma\left(\alpha_{3}+1\right)} \frac{1}{\Gamma\left(\frac{1}{2} \alpha_{1}+2\right) \Gamma\left(\frac{1}{2} \alpha_{2}+1\right) \Gamma\left(\frac{1}{2} \alpha_{3}+1\right)}, \\
& \bar{R}_{I_{1} I_{2} I_{3}}^{s s s}=\frac{\pi}{2}\left(\frac{2}{N}\right)^{\frac{3}{4}} \sigma\left(k_{1}\right) \sigma\left(k_{2}\right) \sigma\left(k_{3}\right) 2^{-\alpha} \frac{1}{\Gamma\left(\frac{1}{2} \alpha+1\right)} \\
& \times \frac{1}{\Gamma\left(\frac{1}{2}\left(\alpha_{1}+1\right)\right) \Gamma\left(\frac{1}{2}\left(\alpha_{2}+1\right)\right) \Gamma\left(\frac{1}{2}\left(\alpha_{3}+1\right)\right)}, \\
& \bar{R}_{I_{1} I_{2} I_{3}}^{s s t}=\frac{\pi}{2}\left(\frac{2}{N}\right)^{\frac{3}{4}} \sigma\left(k_{1}\right) \sigma\left(k_{2}\right) \tau\left(k_{3}\right) 2^{-\alpha} \frac{1}{\Gamma\left(\frac{1}{2} \alpha+2\right)} \\
& \times \frac{\Gamma\left(\alpha_{1}+5\right) \Gamma\left(\alpha_{2}+5\right)}{\Gamma\left(\alpha_{1}+1\right) \Gamma\left(\alpha_{2}+1\right)} \frac{1}{\Gamma\left(\frac{1}{2}\left(\alpha_{1}+3\right)\right) \Gamma\left(\frac{1}{2}\left(\alpha_{2}+3\right)\right) \Gamma\left(\frac{1}{2}\left(\alpha_{3}-1\right)\right)},
\end{aligned}
$$




$$
\begin{aligned}
\bar{R}_{I_{1} I_{2} I_{3}}^{t t s} & =\frac{\pi}{2}\left(\frac{2}{N}\right)^{\frac{3}{4}} \tau\left(k_{1}\right) \tau\left(k_{2}\right) \sigma\left(k_{3}\right) 2^{-\alpha} \frac{\Gamma(\alpha+8)}{\Gamma(\alpha+4)} \frac{1}{\Gamma\left(\frac{1}{2} \alpha+3\right)} \\
& \times \frac{\Gamma\left(\alpha_{3}+5\right) \Gamma\left(\alpha_{3}+11\right)}{\Gamma\left(\alpha_{3}+1\right) \Gamma\left(\alpha_{3}+3\right)} \frac{1}{\Gamma\left(\frac{1}{2}\left(\alpha_{1}+1\right)\right) \Gamma\left(\frac{1}{2}\left(\alpha_{2}+1\right)\right) \Gamma\left(\frac{1}{2}\left(\alpha_{3}+5\right)\right)} \\
\bar{R}_{I_{1} I_{2} I_{3}}^{t t t} & =\frac{\pi}{2}\left(\frac{2}{N}\right)^{\frac{3}{4}} \tau\left(k_{1}\right) \tau\left(k_{2}\right) \tau\left(k_{3}\right) 2^{-\alpha} \frac{\Gamma(\alpha+8) \Gamma(\alpha+14)}{\Gamma(\alpha+4) \Gamma(\alpha+6)} \frac{1}{\Gamma\left(\frac{1}{2} \alpha+4\right)} \\
& \times \frac{\Gamma\left(\alpha_{1}+5\right) \Gamma\left(\alpha_{2}+5\right) \Gamma\left(\alpha_{3}+5\right)}{\Gamma\left(\alpha_{1}+1\right) \Gamma\left(\alpha_{2}+1\right) \Gamma\left(\alpha_{3}+1\right)} \frac{1}{\Gamma\left(\frac{1}{2}\left(\alpha_{1}+3\right)\right) \Gamma\left(\frac{1}{2}\left(\alpha_{2}+3\right)\right) \Gamma\left(\frac{1}{2}\left(\alpha_{3}+3\right)\right)}(3
\end{aligned}
$$

$\underline{\mathrm{AdS}_{5} \times \mathrm{S}_{5}}$

In this case one has $\frac{1}{4 \kappa^{2}}=\frac{N^{2}}{8 \pi^{5}}$ and $\bar{e}=1$. One has

$$
\begin{aligned}
\Delta_{I}^{f} & =k+4, \\
\Delta_{I}^{s} & =k, \\
\Delta_{I}^{t} & =k+8 .
\end{aligned}
$$

Set

$$
\begin{aligned}
\phi(k) & =\left[\frac{k !}{(k+3) !}\right]^{\frac{1}{2}}, \\
\sigma(k) & =k^{\frac{1}{2}}, \\
\tau(k) & =\left[\frac{k !(k+1) !(k+2) !}{(k+5) !(k+6) !(k+7) !}\right]^{\frac{1}{2}} .
\end{aligned}
$$

Then,

$$
\begin{aligned}
\bar{R}_{I_{1} I_{2} I_{3}}^{f f f} & =\frac{1}{N} \phi\left(k_{1}\right) \phi\left(k_{2}\right) \phi\left(k_{3}\right) \frac{\Gamma(\alpha+4)}{\Gamma(\alpha+2)} \frac{\Gamma\left(\alpha_{1}+2\right) \Gamma\left(\alpha_{2}+2\right) \Gamma\left(\alpha_{3}+2\right)}{\Gamma\left(\alpha_{1}+1\right) \Gamma\left(\alpha_{2}+1\right) \Gamma\left(\alpha_{3}+1\right)} \\
\bar{R}_{I_{1} I_{2} I_{3}}^{f f s} & =\frac{1}{N} \phi\left(k_{1}\right) \phi\left(k_{2}\right) \sigma\left(k_{3}\right) \frac{\Gamma\left(\alpha_{3}+3\right) \Gamma\left(\alpha_{3}+4\right)}{\Gamma\left(\alpha_{3}+1\right) \Gamma\left(\alpha_{3}+2\right)} \\
\bar{R}_{I_{1} I_{2} I_{3}}^{f f t} & =\frac{1}{N} \phi\left(k_{1}\right) \phi\left(k_{2}\right) \tau\left(k_{3}\right) \frac{\Gamma(\alpha+5) \Gamma(\alpha+6)}{\Gamma(\alpha+3) \Gamma(\alpha+4)} \\
& \times \frac{\Gamma\left(\alpha_{1}+3\right) \Gamma\left(\alpha_{1}+4\right)}{\Gamma\left(\alpha_{1}+1\right) \Gamma\left(\alpha_{1}+2\right)} \frac{\Gamma\left(\alpha_{2}+3\right) \Gamma\left(\alpha_{2}+4\right)}{\Gamma\left(\alpha_{2}+1\right) \Gamma\left(\alpha_{2}+2\right)} \\
\bar{R}_{I_{1} I_{2} I_{3}}^{f s s} & =\frac{1}{N} \phi\left(k_{1}\right) \sigma\left(k_{2}\right) \sigma\left(k_{3}\right) \frac{\Gamma\left(\alpha_{2}+2\right)}{\Gamma\left(\alpha_{2}+1\right)} \frac{\Gamma\left(\alpha_{3}+2\right)}{\Gamma\left(\alpha_{3}+1\right)} \\
\bar{R}_{I_{1} I_{2} I_{3}}^{f s t} & =\frac{1}{N} \phi\left(k_{1}\right) \sigma\left(k_{2}\right) \tau\left(k_{3}\right) \frac{\Gamma(\alpha+4)}{\Gamma(\alpha+2)}
\end{aligned}
$$




$$
\begin{aligned}
& \times \frac{\Gamma\left(\alpha_{1}+2\right)}{\Gamma\left(\alpha_{1}\right)} \frac{\Gamma\left(\alpha_{2}+5\right) \Gamma\left(\alpha_{2}+6\right)}{\Gamma\left(\alpha_{2}+1\right) \Gamma\left(\alpha_{2}+3\right)} \frac{\Gamma\left(\alpha_{3}\right)}{\Gamma\left(\alpha_{3}+1\right)}, \\
\bar{R}_{I_{1} I_{2} I_{3}}^{f t t} & =\frac{1}{N} \phi\left(k_{1}\right) \tau\left(k_{2}\right) \tau\left(k_{3}\right) \frac{\Gamma(\alpha+7) \Gamma(\alpha+8)}{\Gamma(\alpha+2) \Gamma(\alpha+5)} \\
& \times \frac{\Gamma\left(\alpha_{1}+5\right) \Gamma\left(\alpha_{1}+6\right)}{\Gamma\left(\alpha_{1}\right) \Gamma\left(\alpha_{1}+3\right)} \frac{\Gamma\left(\alpha_{2}+2\right)}{\Gamma\left(\alpha_{2}+1\right)} \frac{\Gamma\left(\alpha_{3}+2\right)}{\Gamma\left(\alpha_{3}+1\right)}, \\
\bar{R}_{I_{1} I_{2} I_{3}}^{s s s} & =\frac{1}{N} \sigma\left(k_{1}\right) \sigma\left(k_{2}\right) \sigma\left(k_{3}\right) \\
\bar{R}_{I_{1} I_{2} I_{3}}^{s s t} & =\frac{1}{N} \sigma\left(k_{1}\right) \sigma\left(k_{2}\right) \tau\left(k_{3}\right) \frac{\Gamma\left(\alpha_{1}+3\right) \Gamma\left(\alpha_{1}+4\right) \Gamma\left(\alpha_{2}+3\right) \Gamma\left(\alpha_{2}+4\right)}{\Gamma\left(\alpha_{1}+1\right) \Gamma\left(\alpha_{1}+2\right) \Gamma\left(\alpha_{2}+1\right) \Gamma\left(\alpha_{2}+2\right)}, \\
\bar{R}_{I_{1} I_{2} I_{3}}^{t s s} & =\frac{1}{N} \tau\left(k_{1}\right) \tau\left(k_{2}\right) \sigma\left(k_{3}\right) \frac{\Gamma(\alpha+5) \Gamma(\alpha+6)}{\Gamma(\alpha+3) \Gamma(\alpha+4)} \frac{\Gamma\left(\alpha_{3}+7\right) \Gamma\left(\alpha_{3}+8\right)}{\Gamma\left(\alpha_{3}+1\right) \Gamma\left(\alpha_{3}+2\right)}, \\
\bar{R}_{I_{1} I_{2} I_{3}}^{t t t} & =\frac{1}{N} \tau\left(k_{1}\right) \tau\left(k_{2}\right) \tau\left(k_{3}\right) \frac{\Gamma(\alpha+9) \Gamma(\alpha+10)}{\Gamma(\alpha+3) \Gamma(\alpha+4)} \\
& \times \frac{\Gamma\left(\alpha_{1}+3\right) \Gamma\left(\alpha_{1}+4\right) \Gamma\left(\alpha_{2}+3\right) \Gamma\left(\alpha_{2}+4\right) \Gamma\left(\alpha_{3}+3\right) \Gamma\left(\alpha_{3}+4\right)}{\Gamma\left(\alpha_{1}+1\right) \Gamma\left(\alpha_{1}+2\right) \Gamma\left(\alpha_{2}+1\right) \Gamma\left(\alpha_{2}+2\right) \Gamma\left(\alpha_{3}+1\right) \Gamma\left(\alpha_{3}+2\right)} .
\end{aligned}
$$

It is useful to remember that the value of the integer $k$ of the operators $\mathcal{O}_{I}^{s}, \mathcal{O}_{I}^{f}, \mathcal{O}_{I}^{t}$ belonging to the same multiplet are related as follows

$$
k^{s}=k, \quad k^{f}=k-2, \quad k^{t}=k-4 .
$$

Some of these results have been obtained by other groups as well. Namely, in the $\mathrm{SCFT}_{4}$ case, the 3-point function of the chiral primary operators eq. $(3.17 \mathrm{~g})$ was produced in the seminal paper [13], while eq. $(3.17 h)$ was contained in [17]. As for $\mathrm{SCFT}_{6}$, eq. $(3.11 \mathrm{~g})$ was calculated in [15], though that result differs for a factor from ours [23]. We have computed in previous works the $s-t$ correlations functions [23,24], extended here above to the full universal sector.

\section{Conclusions}

We have computed in a systematic way the large $N$ limit of the 3-point functions of the scalars belonging to the universal sector which is common to all of the maximal supersymmetric CFT in $d=3,4,6$. The infrared dynamics of the M2, D3 and M5 branes belongs to the universality classes identified by these $\mathrm{SCFT}_{3,4,6}$, and in fact we used the AdS/CFT correspondence to compute some scalar correlation functions in the latter. That constitutes the main result of this paper.

At large $N$, all the operators group into short (also called chiral) supersymmetric multiplets [31-35]. In particular, the operators $\mathcal{O}^{s}$ are the chiral primaries which generate 
all of the other by application of the superconformal algebra [36,37]. In $d=4$ it was shown in [38] by using a superspace approach that the 3-point function of all chiral operators are fixed by a unique superspace conformal invariant up to a single coefficient, which can be easily read off from $\left\langle\mathcal{O}^{s} \mathcal{O}^{s} \mathcal{O}^{s}\right\rangle$. Thus, it should be possible to obtain the correlation functions of the descendants, including the ones listed in section 3, by such superspace techniques, though this may be laborious. A similar strategy has not been worked out for the $\mathrm{SCFT}_{3,6}$, to our knowledge, and it is not known how many independent numbers describe the correlation functions of the chiral multiplets. Investigation of the stress tensor 3 -point functions (belonging to the family identified by the chiral primary with $k=2$ ) show only a single overall coefficient for all of the $\mathrm{SCFT}_{3,6}$ [39], and this may suggest that also in these new cases supersymmetry fixes the correlations function of chiral multiplets up to a constant. It would indeed be interesting to develop a superspace approach and reproduce the descendant correlation functions from the chiral ones. More importantly, it would be nice to investigate their $\frac{1}{N}$ corrections. On the other hand, the general model we used to obtain the scalar 3-point function can still be employed to compute the 4-point functions of the chiral primaries in a systematic way for the $\mathrm{SCFT}_{3,4,6}$. In the light of some results for the super Yang Mills case [20], that might be a formidable task but still within the reach of future research.

\section{A1. Spherical Harmonics}

We describe the $n$-dimensional sphere $S_{n}$ of radius $\rho$ as $S_{n}=\left\{x \in \mathbb{R}^{n+1} \mid x^{2}=\rho^{2}\right\}$.

Since $\mathrm{S}_{n} \subset \mathbb{R}^{n+1}$, we can represent tensor fields on $\mathrm{S}_{n}$ by means of tensor fields on $\mathbb{R}^{n+1}$ of the same type satisfying certain conditions. Since further $S_{n}$ is naturally equipped with the metric induced by the Euclidean metric of $\mathbb{R}^{n+1}$, we need not distinguish covariant and contravariant tensor indices. Specifically, a rank $s$ tensor field $T_{\alpha_{1} \ldots \alpha_{s}}$ on $\mathrm{S}_{n}$ may be viewed as a rank $s$ tensor field $T_{i_{1} \ldots i_{s}}$ defined on a neighborhood of $\mathrm{S}_{n} \subset \mathbb{R}^{n+1}$ with no components normal to $\mathrm{S}_{n}$ :

$$
x_{i} T_{i_{1} \ldots i_{a-1} i i_{a+1} \ldots i_{s}}(x)=0, \quad 1 \leq a \leq s .
$$

In particular, the induced metric $g_{\alpha \beta}$ of $\mathrm{S}_{n}$ is represented by

$$
g_{i j}(x)=\delta_{i j}-\frac{x_{i} x_{j}}{r^{2}}
$$

where $r=\left(x_{i} x_{i}\right)^{\frac{1}{2}}$. Therefore, the contraction of two rank $s$ tensor fields $T_{\alpha_{1} \ldots \alpha_{s}}, U_{\alpha_{1} \ldots \alpha_{s}}$ is given by

$$
T^{\alpha_{1} \ldots \alpha_{s}} U_{\alpha_{1} \ldots \alpha_{s}}=T_{i_{1} \ldots i_{s}} U_{i_{1} \ldots i_{s}} .
$$


For a rank $s$ tensor field $T_{\alpha_{1} \ldots \alpha_{s}}$, the covariant derivative $\nabla_{\alpha} T_{\alpha_{1} \ldots \alpha_{s}}$ is represented by

$$
\nabla_{i} T_{i_{1} \ldots i_{s}}(x)=\left(\partial_{i}-\frac{x_{i}}{r} \partial_{r}\right) T_{i_{1} \ldots i_{s}}(x)+\sum_{a=1}^{s} \frac{x_{i_{a}}}{r^{2}} T_{i_{1} \ldots i_{a-1} i i_{a+1} \ldots i_{s}}(x)
$$

where $\partial_{r}=\partial / \partial r$.

For any function $F$ on $\mathrm{S}_{n}$, the integral of $F$ on $\mathrm{S}_{n}$ is given by

$$
\int_{\mathrm{S}_{n}} d^{n} \operatorname{vol} F=\left.\int_{\mathrm{S}_{n}} r^{n} d \Omega_{n}(x) F(x)\right|_{r=\rho}
$$

where $d \Omega_{n}(x)$ is the standard $n$ dimensional volume on $\mathrm{S}_{n}$ defined as usual by $d^{n+1} x=$ $r^{n} d r d \Omega_{n}(x)$.

Using the above integration formula, one can provide the space of rank $s$ tensor fields with a Hilbert space structure in obvious fashion.

Consider the rank $s$ tensor fields

$$
Y_{I i_{1} \ldots i_{s}}^{(s)}(x)=\mathcal{Y}_{I i_{1} \ldots i_{s} ; j_{1} \ldots j_{k}}^{(s)} r^{-k} x_{j_{1}} \ldots x_{j_{k}},
$$

where the $\mathcal{Y}_{I i_{1} \ldots i_{s} ; j_{1} \ldots j_{k}}^{(s)}$ are constants satisfying the following conditions:

i) $\mathcal{Y}_{I i_{1} \ldots i_{s} ; j_{1} \ldots j_{k}}^{(s)}$ is symmetric and traceless in $i_{1}, \ldots, i_{s}$;

ii) $\mathcal{Y}_{I i_{1} \ldots i_{s} ; j_{1} \ldots j_{k}}^{(s)}$ is symmetric and traceless in $j_{1}, \ldots, j_{k}$;

iii) the following relation holds

$$
\mathcal{Y}_{I i_{1} \ldots i_{s-1}\left\{i_{s} ; j_{1} \ldots j_{k}\right\}}^{(s)}=0
$$

where $\{\ldots\}$ denotes total symmetrization.

The index $I$ labels the different choices of the constants $\mathcal{Y}_{I i_{1} \ldots i_{s} ; j_{1} \ldots j_{k}}^{(s)}$. Note that $Y_{I i_{1} \ldots i_{s}}^{(s)}$ is characterized by the non negative integer $k$, which may be thought of as a function of the label $I$. It can be shown that, if $Y_{I i_{1} \ldots i_{s}}^{(s)} \neq 0$, then necessarily $k \geq s$.

Then, the $Y_{I i_{1} \ldots i_{s}}^{(s)}$ are symmetric traceless rank $s$ tensor fields on $\mathrm{S}_{n}$. Further, they are divergenceless (transversal):

$$
\nabla_{i} Y_{I i i_{2} \ldots i_{s}}^{(s)}=0
$$

Finally, the $Y_{I i_{1} \ldots i_{s}}^{(s)}$ are eigenfields of the Laplacian:

$$
-\nabla_{i} \nabla_{i} Y_{I i_{1} \ldots i_{s}}^{(s)}=\frac{1}{r^{2}}\left[(k(k+n-1)-s] Y_{I i_{1} \ldots i_{s}}^{(s)}\right.
$$

If, for fixed $s$, we choose a maximal set of constants $\mathcal{Y}_{I i_{1} \ldots i_{s} ; j_{1} \ldots j_{k}}^{(s)}$ such that

$$
\mathcal{Y}_{I i_{1} \ldots i_{s} ; j_{1} \ldots j_{k}}^{(s)} \mathcal{Y}_{J i_{1} \ldots i_{s} ; j_{1} \ldots j_{k}}^{(s)}=\delta_{I J}
$$


for all $k$, then, the tensor fields $\left\{Y_{I i_{1} \ldots i_{s}}^{(s)} \mid I\right\}$ provide an orthogonal basis of the space of divergenceless symmetric traceless rank $s$ tensor fields on $\mathrm{S}_{n}$.

In concrete applications, one has to compute the integrals of scalars formed by contraction of several tensor spherical harmonics $Y^{\left(s_{\ell}\right)}$ and their covariant derivatives. This can be done by a systematic use of the formula

$$
\begin{aligned}
\left.\int_{S^{n}} r^{n} d \Omega_{n}(x) r^{-2 m} x^{i_{1}} \cdots x^{i_{2 m}}\right|_{r=\rho} & =\rho^{n} \omega_{n} \frac{(n-1) ! !}{(2 m+n-1) ! !} \\
& \times(\text { all possible Wick contractions })
\end{aligned}
$$

where "all possible Wick contractions" are given by the sum of $(2 m-1)$ !! terms obtained by using $\left\langle r^{-2} x^{i} x^{j}\right\rangle=\delta^{i j}$ as elementary Wick contraction and $\omega_{n}$ is the volume of the unit sphere

$$
\omega_{n}=\frac{2 \pi^{\frac{n+1}{2}}}{\Gamma\left(\frac{n+1}{2}\right)} .
$$

The results are always expressible in terms of suitable contractions of the coefficients $\mathcal{Y}_{I i_{1} \ldots i_{s_{\ell}} ; j_{1} \ldots j_{k_{\ell}}}^{\left(s_{\ell}\right)}$ generically denoted as $\left\langle\prod_{\ell} \mathcal{Y}_{I_{\ell}}^{\left(s_{\ell}\right)}\right\rangle$ and certain numerical functions of the $k_{\ell}$. For two and three tensor spherical harmonics, the only such functions are

$$
\begin{aligned}
z_{I} & =\omega_{n} \frac{(n-1) ! ! k !}{(2 k+n-1) ! !} \\
a_{I_{1} I_{2} I_{3}} & =\omega_{n} \frac{(n-1) ! !}{(2 \alpha+n-1) ! !} \frac{k_{1} ! k_{2} ! k_{3} !}{\alpha_{1} ! \alpha_{2} ! \alpha_{3} !} .
\end{aligned}
$$

where

$$
\begin{gathered}
\alpha_{1}=\frac{1}{2}\left(k_{2}+k_{3}-k_{1}\right), \quad \alpha_{2}=\frac{1}{2}\left(k_{3}+k_{1}-k_{2}\right), \quad \alpha_{3}=\frac{1}{2}\left(k_{1}+k_{2}-k_{3}\right), \\
\alpha=\frac{1}{2}\left(k_{1}+k_{2}+k_{3}\right) .
\end{gathered}
$$

In this paper, $\rho=\bar{e}^{-1}$. Further, we consider only $s=0,2$. We set $\mathcal{C}_{I ; j_{1} \ldots j_{k}}=\mathcal{Y}_{I ; j_{1} \ldots j_{k}}^{(0)}$ and $\mathcal{T}_{I i_{1} i_{2} ; j_{1} \ldots j_{k}}=\mathcal{Y}_{I i_{1} i_{2} ; j_{1} \ldots j_{k}}^{(2)}$.

The main contractions are the following. $\left\langle\mathcal{C}_{I_{1}} \mathcal{C}_{I_{2}} \mathcal{C}_{I_{3}}\right\rangle$ denotes the unique $S O(n+1)$ scalar contraction of three tensors $\mathcal{C}_{I i_{1} \ldots i_{k}}$ and in a similar fashion we define

$$
\begin{aligned}
\left\langle\mathcal{T}_{I_{1}} \mathcal{C}_{I_{2}} \mathcal{C}_{I_{3}}\right\rangle & =\left\langle\mathcal{T}_{I_{1} a b} \mathcal{C}_{I_{2} ; a} \mathcal{C}_{I_{3} ; b}\right\rangle \\
\left\langle\mathcal{T}_{I_{1}} \mathcal{T}_{I_{2}} \mathcal{C}_{I_{3}}\right\rangle & =\left\langle\mathcal{T}_{I_{1} a b} \mathcal{T}_{I_{2} a b} \mathcal{C}_{I_{3}}\right\rangle \\
\left\langle\mathcal{T}_{I_{1}} \mathcal{T}_{I_{2}} \mathcal{T}_{I_{3}}\right\rangle & =4\left\langle\mathcal{T}_{I_{1} a b} \mathcal{T}_{I_{2} b c} \mathcal{T}_{I_{3} c a}\right\rangle \\
& +\sum_{\text {c.p. } 123} \alpha_{1}\left(2\left\langle\mathcal{T}_{I_{1} a b} \mathcal{T}_{I_{2} a c ; d} \mathcal{T}_{I_{3} b d ; c}\right\rangle+\left\langle\mathcal{T}_{I_{1} a b} \mathcal{T}_{I_{2} c d ; a} \mathcal{T}_{I_{3} c d ; b}\right\rangle\right)
\end{aligned}
$$


where on the right hand side one takes the unique contraction of the hidden indices, and where the notation c.p.123 stands for cyclic permutations of 123 .

\section{A2. Auxiliary functions}

Here is the complete list of the auxiliary functions $F$ appearing in $(2.4 a)-(2.4 j)$.

$$
\begin{aligned}
& F^{f s s}=(D-2) \alpha_{2} \alpha_{3} \\
& F^{f t t}=(D-2) \alpha_{2} \alpha_{3} \\
& F^{f s t}=(D-2) \alpha_{1}(\alpha+1+p) \\
& F^{s s s}=(1+p)(D-3-p)\left(\alpha_{1}^{2} \alpha_{2}+\alpha_{2}{ }^{2} \alpha_{1}+\alpha_{2}{ }^{2} \alpha_{3}+\alpha_{3}{ }^{2} \alpha_{2}+\alpha_{3}{ }^{2} \alpha_{1}+\alpha_{1}{ }^{2} \alpha_{3}\right) \\
& +\left(3 D-8+(2 D-8) p-2 p^{2}\right) \alpha_{1} \alpha_{2} \alpha_{3} \\
& +(1+p)\left(-\frac{1}{2} D+2+p\right)\left(\alpha_{1} \alpha_{2}+\alpha_{2} \alpha_{3}+\alpha_{3} \alpha_{1}\right) \\
& F^{s s t}=(1+p)(D-3-p)\left(\alpha_{1}^{2} \alpha_{3}+\alpha_{3}^{2} \alpha_{1}+\alpha_{2}{ }^{2} \alpha_{3}+\alpha_{3}{ }^{2} \alpha_{2}\right) \\
& +\left(-1+(D-4) p-p^{2}\right)\left(\alpha_{1}^{2} \alpha_{2}+\alpha_{2}^{2} \alpha_{1}\right) \\
& +\left(D-4+(2 D-8) p-2 p^{2}\right) \alpha_{1} \alpha_{2} \alpha_{3} \\
& +(1+p) p(D-3-p)\left(\alpha_{1} \alpha_{3}+\alpha_{2} \alpha_{3}\right) \\
& +(1+p)\left(-D+2+(D-3) p-p^{2}\right) \alpha_{1} \alpha_{2} \\
& +(1+p)(D-3-p)\left(-\alpha_{1}^{2}-\alpha_{2}^{2}+(1+p) \alpha_{3}^{2}\right) \\
& +(1+p)^{2}(-D+3+p)\left(\alpha_{1}+\alpha_{2}+\alpha_{3}\right) \\
& F^{t t s}=(1+p)(D-3-p)\left(\alpha_{1}{ }^{2} \alpha_{3}+\alpha_{3}{ }^{2} \alpha_{1}+\alpha_{2}{ }^{2} \alpha_{3}+\alpha_{3}{ }^{2} \alpha_{2}\right) \\
& +\left(-1+(D-4) p-p^{2}\right)\left(\alpha_{1}^{2} \alpha_{2}+\alpha_{2}^{2} \alpha_{1}\right) \\
& +\left(D-4+(2 D-8) p-2 p^{2}\right) \alpha_{1} \alpha_{2} \alpha_{3} \\
& +(1+p)\left(\frac{5}{2} D-8+(2 D-9) p-2 p^{2}\right)\left(\alpha_{1} \alpha_{3}+\alpha_{2} \alpha_{3}\right) \\
& +(1+p)\left(\frac{3}{2} D-6+(2 D-9) p-2 p^{2}\right) \alpha_{1} \alpha_{2} \\
& +(1+p)\left(\frac{3}{2} D-5+(D-5) p-p^{2}\right)\left(\alpha_{1}^{2}+\alpha_{2}^{2}\right) \\
& +(1+p)^{2}\left(\frac{3}{2} D-5+(D-5) p-p^{2}\right)\left(\alpha_{1}+\alpha_{2}\right)
\end{aligned}
$$




$$
\begin{aligned}
F^{t t t}=(1 & +p)(D-3-p)\left(\alpha_{1}{ }^{2} \alpha_{2}+\alpha_{2}{ }^{2} \alpha_{1}+\alpha_{2}{ }^{2} \alpha_{3}+\alpha_{3}{ }^{2} \alpha_{2}+\alpha_{3}{ }^{2} \alpha_{1}+\alpha_{1}{ }^{2} \alpha_{3}\right) \\
& +\left(3 D-8+(2 D-8) p-2 p^{2}\right) \alpha_{1} \alpha_{2} \alpha_{3} \\
& +(1+p)\left(4 D-13+(3 D-14) p-3 p^{2}\right)\left(\alpha_{1} \alpha_{2}+\alpha_{2} \alpha_{3}+\alpha_{3} \alpha_{1}\right) \\
& +(1+p)^{2}\left(D-\frac{7}{2}-p\right)\left(\alpha_{1}^{2}+\alpha_{2}{ }^{2}+\alpha_{3}^{2}\right) \\
& +(1+p)^{2}\left(3 D-11+\left(2 D-\frac{21}{2}\right) p-2 p^{2}\right)\left(\alpha_{1}+\alpha_{2}+\alpha_{3}\right) \\
& +(2+p)(1+p)^{3}(D-4-p)
\end{aligned}
$$




\section{REFERENCES}

[1] N. Seiberg, "Notes on theories with 16 supercharges", Nucl. Phys. Proc. Suppl. 67 (1998) 158, hep-th/9705117.

[2] E. Witten, "Bound states of strings and p-branes", Nucl. Phys. B460 (1996) 335, hep-th/9510135.

[3] G. W. Gibbons and P. K. Townsend, "Vacuum interpolation in supergravity via super p-branes", Phys. Rev. Lett. 71 (1993) 3754, hep-th/9307049.

[4] D. M. Kaplan and J. Michelson, "Zero modes for the $\mathrm{D}=11$ membrane and five-brane", Phys. Rev. D53 (1996) 3474, hep-th/9510053.

[5] E. Bergshoeff, E. Sezgin and P. K. Townsend, "Supermembranes and eleven dimensional supergravity", Phys. Lett. B189 (1987) 75.

[6] E. Witten, "Some comments on string dynamics", hep-th/9507121.

[7] A. Strominger, "Open p-branes", Phys. Lett. B383 (1996) 44, hep-th/9512059.

[8] E. Witten, "Five-branes and M-theory on an orbifold", Nucl. Phys. B463 (1996) 383, hep-th/9512219.

[9] J. Maldacena, "The large $N$ limit of superconformal field theories and supergravity", Adv. Theor. Math. Phys. 2 (1998) 231, hep-th/9711200.

[10] S. S. Gubser, I. R. Klebanov and A. M. Polyakov, "Gauge theory correlators from non-critical string theory", Phys. Lett. B428 (1998) 105, hep-th/9802109.

[11] E. Witten, "Anti-de Sitter space and holography", Adv. Theor. Math. Phys. 2 (1998) 253 , hep-th/9802150.

[12] O. Aharony, S. S. Gubser, J. Maldacena, H. Ooguri and Y. Oz, "Large $N$ field theories, string theory and gravity", Phys. Rept. 323 (2000) 183, hep-th/9905111.

[13] S. Lee, S. Minwalla, M. Rangamani and N. Seiberg, "Three-point functions of chiral operators in $D=4, \mathcal{N}=4 \mathrm{SYM}$ at Large $N "$, Adv. Theor. Math. Phys. 2 (1998) 697, hep-th/9806074.

[14] G. Arutyunov and S. Frolov, "Three-point Green function of the stress-energy tensor in the AdS/CFT correspondence", Phys. Rev. D60 (1999) 026004, hep-th/9901121.

[15] R. Corrado, B. Florea and R. McNees, "Correlation functions of operators and Wilson surfaces in the $d=6,(0,2)$ theory in the large $N$ limit", Phys. Rev. D60 (1999) 085011, hep-th/9902153.

[16] E. D'Hoker, D. Z. Freedman, S. D. Mathur, A. Matusis and L. Rastelli, "Graviton exchange and complete 4-point functions in the AdS/CFT correspondence", Nucl. Phys. B562 (1999) 353, hep-th/9903196.

[17] G. Arutyunov and S. Frolov, "Some cubic couplings in type IIB supergravity on $A d S_{5} \times$ $S^{5}$ and three-point functions in $\mathrm{SYM}_{4}$ at large $N$ ", Phys. Rev. D61 (2000) 064009, hep-th/9907085. 
[18] S. Lee, "AdS(5)/CFT(4) four-point functions of chiral primary operators: cubic vertices", Nucl. Phys. B563 (1999) 349, hep-th/9907108.

[19] E. D'Hoker, D. Z. Freedman, S. D. Mathur, A. Matusis and L. Rastelli, "Extremal correlators in the AdS/CFT correspondence", hep-th/9908160.

[20] G. Arutyunov and S. Frolov, "Four-point functions of lowest weight CPOs in $\mathcal{N}=4$ $\mathrm{SYM}_{4}$ in supergravity approximation", hep-th/0002170.

[21] R. Manvelian and A. C. Petkou, "A note on R-currents and trace anomalies in the $(2,0)$ tensor multiplet in $d=6$ AdS/CFT correspondence", hep-th/0003017.

[22] F. Bastianelli and R. Zucchini, "Bosonic quadratic actions for 11D supergravity on $\mathrm{AdS}_{7 / 4} \times \mathrm{S}_{4 / 7} "$, Class. Quant. Grav. 16 (1999) 3673, hep-th/9903161.

[23] F. Bastianelli and R. Zucchini, "Three point functions of chiral primary operators in $d=3, \mathcal{N}=8$ and $d=6, \mathcal{N}=(2,0)$ SCFT at large $N "$, Phys. Lett. B467 (1999) 61, hep-th/9907047.

[24] F. Bastianelli and R. Zucchini, "Three point functions for a class of chiral operators in maximally supersymmetric CFT at large $N "$, hep-th/9909179.

[25] K. Intriligator, "Bonus symmetries of $\mathcal{N}=4$ super-Yang-Mills correlation functions via AdS duality", Nucl. Phys. B551 (1999) 575, hep-th/9811047.

[26] K. Intriligator, "Maximally supersymmetric RG flows and AdS duality," hep-th/ 9909082.

[27] P. Freund and M. Rubin, "Dynamics of dimensional reduction", Phys. Lett. B97 (1980), 233.

[28] P. van Nieuwenhuizen, "The complete mass spectrum of $d=11$ supergravity compactified on $S^{4}$ and a general mass formula for arbitrary cosets $M_{4}$ ", Class. Quantum Grav. 2 (1985), 1.

[29] W. Muck and K. S. Viswanathan, "Conformal field theory correlators from classical scalar field theory on AdS(d+1)", Phys. Rev. D58 (1998) 041901, hep-th/9804035.

[30] D. Z. Freedman, S. D. Mathur, A. Matusis and L. Rastelli, "Correlation functions in the CFT $(d) / \operatorname{AdS}(d+1)$ correspondence", Nucl. Phys. B546 (1999) 96, hep-th/ 9804058 .

[31] M. Gunaydin, P. van Nieuwenhuizen and N. P. Warner, "General construction of the unitary representations of anti-De Sitter superalgebras and the spectrum of the $S^{4}$ compactification of eleven-dimensional supergravity", Nucl. Phys. B255 (1985) 63.

[32] M. Gunaydin and N. Marcus, "The spectrum of the $S^{5}$ compactification of the chiral $N=2, D=10$ supergravity and the unitary supermultiplets of $U(2,2 / 4)$ ", Class. Quant. Grav. 2 (1985) L11. 
[33] M. Gunaydin, D. Minic and M. Zagermann, "Novel supermultiplets of $S U(2,2 \mid 4)$ and the AdS(5)/CFT(4) duality", Nucl. Phys. B544 (1999) 737, hep-th/9810226; "4D doubleton conformal theories, CPT and II B string on $\operatorname{AdS}(5)$ x S(5)", Nucl. Phys. B534 (1998) 96 hep-th/9806042.

[34] S. Ferrara and E. Sokatchev, "Representations of $(1,0)$ and $(2,0)$ superconformal algebras in six dimensions: Massless and short superfields", hep-th/0001178; "Short representations of $\mathrm{SU}(2,2 / \mathrm{N})$ and harmonic superspace analyticity", hep-th/9912168.

[35] L. Andrianopoli, S. Ferrara, E. Sokatchev and B. Zupnik, "Shortening of primary operators in N-extended SCFT(4) and harmonic-superspace analyticity", hep-th/ 9912007.

[36] W. Nahm, "Supersymmetries and their representations", Nucl. Phys. B135 (1978) 149.

[37] V. K. Dobrev and V. B. Petkova, "All positive energy unitary irreducible representations of extended conformal supersymmetry", Phys. Lett. B162 (1985) 127; "Group theoretical approach to extended conformal supersymmetry: function space realizations and invariant differential operators", Fortsch. Phys. 35 (1987) 537.

[38] P. S. Howe, E. Sokatchev and P. C. West, "Three point functions in $N=4$ YangMills", Phys. Lett. B444 (1998) 341, hep-th/9808162.

[39] F. Bastianelli, S. Frolov and A. A. Tseytlin, "Three-point correlators of stress tensors in maximally-supersymmetric conformal theories in $\mathrm{d}=3$ and $\mathrm{d}=6$ ", hepth/9911135; "Conformal anomaly of $(2,0)$ tensor multiplet in six dimensions and AdS/CFT correspondence", JHEP 02 (2000) 013, hep-th/0001041. 